\title{
Admission control and resource allocation strategies for IEEE 802.15.5
}

\author{
Samar Sindian ${ }^{1,2^{*}}$, Matthieu Crussière', Jean-François Hélard ${ }^{1}$, Abed Ellatif Samhat ${ }^{3}$ and Ayman Khalil ${ }^{1,2}$
}

\begin{abstract}
In this paper, a novel two-tier mechanism for the access control and resource allocation of a two-hop high data rate IEEE 802.15.5 network is proposed. One of the main contributions in this proposal is the use of a dynamic superframe size in the IEEE 802.15.5. Additionally, the ideas of using a superframe utilization threshold and a channel time allocation period (CTAP) utilization threshold are incorporated, with the aim of allowing more devices to access the shared superframe. Using these thresholds, two new hop-1 resource allocation algorithms are described; CTAP utilization threshold-based resource allocation algorithm (CTRA) and superframe utilization threshold-based resource allocation algorithm (STRA). These algorithms control the access of the hop-1 Mesh PicoNet Coordinators (MPNCs) and Mesh DEVices (MDEVs) to the channel time resources in the superframe. Furthermore, an algorithm for the distribution of the hop-1 MPNCs' resources among the real-time (RT) and non-real-time (NRT) MDEVs at hop-2 is introduced. In this mechanism, a new flag metric is applied to provide higher priorities to MPNCs over MDEVs in the hop-1 algorithms and to the RT MDEVs over the NRT MDEVs in the hop-2 algorithm. Simulation results show the superiority of the proposed mechanism over others by providing a higher satisfaction factor and higher fairness among the competing devices at both hop-1 and hop-2 of the IEEE 802.15.5.
\end{abstract}

Keywords: IEEE 802.15.5; High data rate WPAN; Resource allocation; Fairness; Dynamic superframe

\section{Introduction}

A new high data rate (HR) wireless personal area network (WPAN) standard has been developed by IEEE 802.15.3 task group (TG) [1]. The IEEE 802.15.3 standard is designed to provide low complexity, low cost, low power consumption, and high data rate wireless connectivity among portable consumer electronics and communication devices. HR WPAN is expected to play a crucial role in the formation of home area networks and is targeted to support high-end multimedia applications with high throughput requirements. However, HR WPAN has a limited operating range with a single-hop communication between devices. In order to extend this range and enable multi-hop communications, the mesh networking approach is utilized. A HR WPAN mesh network can be formed by interconnecting simultaneously operating piconets (SOPs). Hence, various problems may arise including inter-piconet interferences, beacon collisions,

\footnotetext{
*Correspondence: sindian_s@hotmail.com

${ }^{1}$ INSA de Rennes, IETR, UMR 6164, F-35708 Rennes, France

${ }^{2}$ CCE Department, Faculty of Engineering, IUL, 30014 Khaldeh, Lebanon

Full list of author information is available at the end of the article
}

throughput limitations, channel resource reservation collisions, etc. Different works [2-6] investigate the resource allocation mechanisms and propose solutions to overcome these limitations while trying to extend the coverage area of an IEEE 802.15.3-based WPAN. A detailed description of these works is presented in [7].

However, drawbacks in terms of system coverage and device connectivity are still evident despite the solutions presented in the works stated above. Consequently, and in order to extend the single-hop range of HR WPANs to multiple hops, the IEEE 802.15.5 standard was developed in 2009 to define the necessary specifications for HR WPANs operation in a mesh configuration [8]. The IEEE 802.15.5 [9] network is dynamically self-organized and self-configured, i.e., the nodes in the network automatically establish and maintain mesh connectivity among themselves. IEEE 802.15.5 provides the architectural framework enabling WPAN devices to promote interoperable, stable, and scalable wireless mesh topologies and, if needed, to provide the amendment text to the current WPAN standards that is required to implement this recommended practice [10]. 
In the case of IEEE 802.15.5, multiple WPAN clusters compete for channel time in a shared superframe. Therefore, it is essential to determine the channel time requirements of each cluster with a certain number of devices and to determine how these clusters can compete to the shared channel time.

The work in [11] presents a distributed resource allocation scheme for meshed WPANs based on a utility function. In this paper, a novel centralized scheme for resource allocation in a two-hop IEEE 802.15.5 based on the centralized approach described in [12] is proposed. One of the main contributions in this proposal is the introduction of the notion of dynamic superframe size to the IEEE 802.15.5. This consequently leads to a much more efficient use of the superframe as it will be shown later by results. Additionally, the channel time requests (CTRqs) of both the hop-1 Mesh DEVices (MDEVs) and Mesh PicoNet Coordinators (MPNCs) are taken into consideration. The latter are assigned higher priority and larger channel time allocations (CTAs) providing them with sufficient channel time resources for serving their clusters. The notion of utilization thresholds, which enables the allocation of variable CTA sizes for prompting fairness, is also introduced. Consequently, more devices are granted access to channel time resources but with smaller sizes. In this context, two types of utilization thresholds are proposed in order to be applied in two different hop-1 algorithms. Additionally, a mechanism for distributing the hop-1 MPNC resources among their hop-2 devices is proposed. Moreover, service differentiation is applied by providing higher priority and larger CTA allocations for RT over NRT traffic requests at hop-2. This proposal shows higher satisfaction and fairness by the introduction of a priority flag metric and by taking the devices' rejected requests into account.

The rest of this paper is organized as follows. Section II briefly describes the works related to resource allocation in IEEE 802.15.5. Section III introduces the IEEE 802.15.5 WPAN architecture. In Section IV, the standard resource allocation mechanism is described. In Section V, the new notions to be incorporated into the proposed mechanism are presented. This is followed by the presentation of the different IEEE 802.15.5 hop-1 and hop-2 resource allocation mechanisms in Section VI. In Section VII, the simulation results of the different mechanisms are presented. Finally, Section VIII concludes the paper.

\section{Related work}

Under the IEEE 802.15.5 umbrella, few research works $[11,12]$ investigating the resource allocation mechanisms for the hop-1 of HR IEEE 802.15.5 have been conducted. But to the readers' knowledge, no work has already studied a resource allocation and admission control mechanism at the IEEE 802.15.5 hop-2. Additionally, no work has introduced the notion of service differentiation among the real-time (RT) and non-real-time (NRT) devices in IEEE 802.15.5.

In [11], a distributed algorithm which calculates a fair share in the superframe for each requesting MPNC is proposed. It considers a cooperative approach in which the utility for each ref-MPNC is maximized in a way that the other requesting MPNCs benefit as well. However, the algorithm is based on the individual requests for each flow by the MPNC. The distributed allocation, although having some merits, has some drawbacks. In [9] and [11], as the number of devices increases in each cluster and also in the presence of a dense meshed WPAN with a high number of MPNCs, the probability of channel time request conflict increases. Additionally, due to the lack of an appropriate mechanism to determine a fair share for each MPNC, unfairness is likely in the reservations by the MPNCs in the mentioned distributed mechanisms.

The most relevant work for resource allocation in meshed WPANs is given in [12], where the resource allocation is done on a centralized basis for a two-hop neighborhood. In the centralized approach, one MPNC among each neighborhood of MPNCs which share a common beacon period (BP) in the superframe is selected as the reference MPNC (ref-MPNC) for the other MPNCs to synchronize their time slots with. In the centralized approach, the ref-MPNC keeps track of the time reservations in the superframe up to two hops and therefore assuring that there are no conflicts in channel time reservation. Another feature of the approach in [12] is that the channel time is reserved in bulk by the MPNCs rather than a request by request basis to reduce the amount of control overhead. The idea behind bulk reservation is to join a group of channel time requests in one large request, thus reducing the possibility of CTA conflicts in case of higher device density. The approach in [12] studies the satisfaction and fairness among the MPNCs at hop-1 of the IEEE 802.15.5. In spite of the advantages of this centralized approach over the distributed one, it does not take into consideration the channel time requirements of neither the hop-1 MDEVs in the ref-MPNC cluster nor the hop-2 MDEVs in the hop-1 MPNCs clusters. Moreover, it considers the fixed maximum superframe size $(65,535 \mu \mathrm{s})$ according to the IEEE 802.15.3 [1] which could be inefficiently utilized in case of low-loaded networks. Additionally, each MPNC estimates its channel requirements based on a history of previous flows. Therefore, inefficient superframe utilization can occur because of incorrect channel time estimation for a bulk reservation by each MPNC due to either overestimation or underestimation of the channel time requirement of a cluster. 


\section{Mesh WPAN based on the IEEE 802.15.5 standard} The recommendations for operation in a mesh configuration for WPANs are given in the IEEE 802.15.5 standard [9]. This standard has two parts in which one part defines necessary specifications for low data rate (LR) WPANs [13] and the other part for HR WPANs. The mesh-related functions and services reside over the IEEE 802.15.3 MAC sublayer as shown in Figure 1. Providing multi-hop services necessitates a slight amendment of the underlying MAC sublayer in the reference model.

The LR WPAN provides data rate of about 0.25 Mbps, which is not convenient to support high rate or real-time multimedia traffic. The work in [14] presents a new synchronization approach 'High-Performance Synchronization Algorithm for wireless mesh sensor networks (HIPESYN)', which is adapted to the LR IEEE 802.15.5 standard for synchronous communications. However, because of the low data rate provided by the LR WPAN, our work focuses on the HR WPAN which provides a high data transmission of $55 \mathrm{Mbps}$ that is suitable for supporting RT applications.

\subsection{IEEE 802.15.5 architecture}

The IEEE 802.15.5 Recommended Practice is a member of the WPAN standard family. It is developed based on IEEE 802.15.3-2003/2005 MAC/PHY layer specifications [10]. The architectural framework of the HR WPAN mesh is to allow multiple MPNCs and MDEVs to participate in communications across multiple piconets. The communication between MDEVs and MPNCs of different piconets is called inter-PAN communication while communication between devices in the same piconet is called intra-PAN communication (Figure 2). Additionally, since all the MPNCs could be connected to each other using the mesh topology, there is no single point of failure in the mesh network. Moreover, IEEE 802.15.5 enables the MAC to handle multiple connections at a given time using different channels.

\subsection{IEEE 802.15.5 superframe}

A MPNC manages the synchronization and controls the data traffic of the system. In a meshed WPAN, communications between MPNCs and within their piconets take place in a shared superframe. The superframe can be used for transmission of beacons from MPNCs and data communication. It is composed of three main parts (Figure 3):

- The beacon period: the beacon (Figure 4) is transmitted periodically by a MPNC to provide information about neighbor reservations and provide timing information that aids in synchronization. This helps in avoiding beacon reservation conflicts.

- The channel access period (CAP) is carrier sense multiple access with collision avoidance (CSMA/CA) based and it can be used for sending association requests and other commands. The CAP is shared among the MPNCs.

- The channel time allocation period (CTAP) is time division multiple access (TDMA) based. It is divided into equal length medium access slots (MASs) used to address medium reservations between MPNCs. A MAS is composed of CTAs that are used by devices to exchange data connections. The smallest channel time unit is referred to as time unit (TU). A TU is a unit of time of the CTA with a resolution of $1 \mu$ s to make the allocation of time easier for the MPNC.

\section{IEEE 802.15.5 standard resource allocation mechanism}

Before allocating the channel time resources, a device calculates the necessary number of TUs per superframe. The device then indicates the minimum and the desired number of TUs it requires through a CTRq command (Figure 5) to the MPNC. The MPNC after getting the CTRq from the device checks the available capacity of the superframe, and if suitable, it sends a channel time response command (CTRp) (Figure 6). Using the CTRp, a MPNC indicates to the requesting device the available number of TUs and also notifies the neighbor MPNCs of the new reservation.

The IEEE 802.15.5 standard in [9] identifies that the MPNCs which are in a single-hop transmission range of each other need to synchronize their boundaries to a common ref-MPNC to avoid time slot overlaps due to synchronization issues. The procedure given in [9] for time reservation by a MPNC for intra-PAN flows is

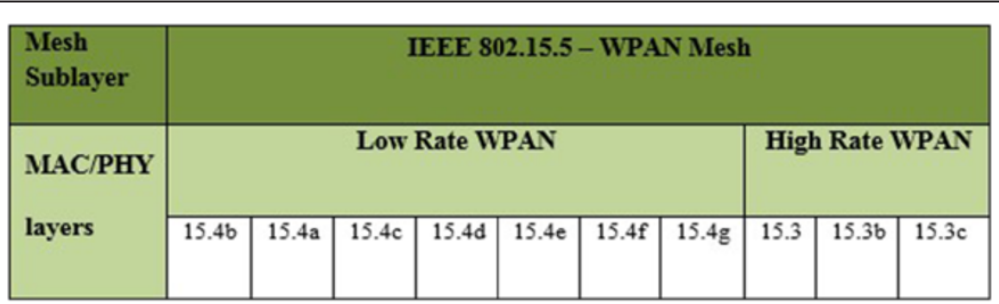

Figure 1 IEEE 802.15.5 layers. 


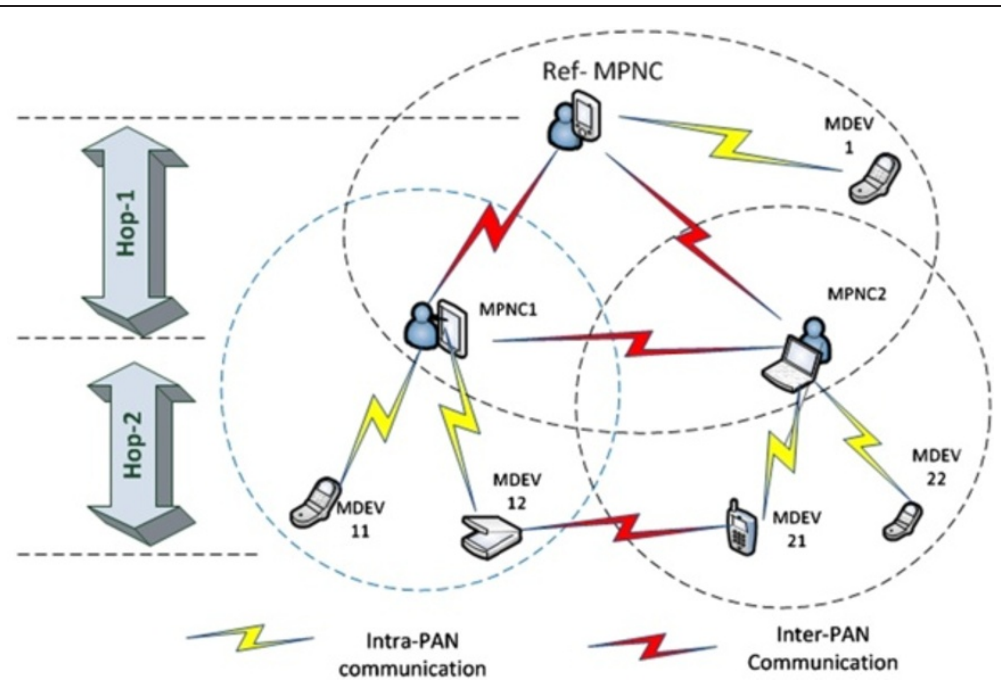

Figure 2 Two-hop IEEE 802.15.5 mesh configuration.

different from inter-PAN flows. But in both cases, if the MPNC reserves channel time in the superframe, it indicates the time reserved via a CTA status information element (IE) and then via a CTA IE in its beacon frame (Figure 4). The other MPNCs, upon checking these IEs, check if the reserved time conflicts with any of the time slots already reserved by them. In case there is a conflict, the other MPNCs can raise an objection IE in their beacon and the MPNC which reserved the time can shift the reserved time slots to another location on the superframe and then wait for an objection again. If there is no objection raised, the MPNC finalizes the reserved time. Since this mechanism is distributed, thus as the number of devices increases in each piconet of a dense meshed WPAN with a higher number of MPNCs, the probability of channel time request conflict increases. Furthermore, the distributed superframe sharing can cause fairness issues because there is no mechanism to determine the requirement of each MPNC and monitor a fair allocation to each MPNC. These are the main drawbacks of the resource allocation mechanism of the IEEE 802.15.5 standard.

\section{IEEE 802.15.5 proposed admission control schemes}

If a MPNC allocates the channel time to a device based on the available time in the superframe only, then most of the devices might not get a fair share of the superframe. While allocating the channel time, the MPNC should ensure an approach such that the maximum number of devices in the piconet gets a fair share of the superframe time based on their requirements.

With the novel proposed admission control approach, a centralized two-tier mechanism for coordinating the resource allocation algorithms of both the hop-1 (between the ref-MPNC and its member hop-1 MDEVs/ MPNCs) and hop-2 (between the hop-1 MPNCs and their member hop-2 RT/NRT MDEVs) of the IEEE 802.15.5 is carried out. With this scheme, the ref-MPNC is used for allocating the channel time to each requesting device at hop-1. Some of the introduced notions are used by both hop- 1 and hop- 2 access mechanisms, namely, bulk channel time requests and flag metric notions. Other notions are introduced only to the hop-1 resource allocation mechanism, namely dynamic superframe

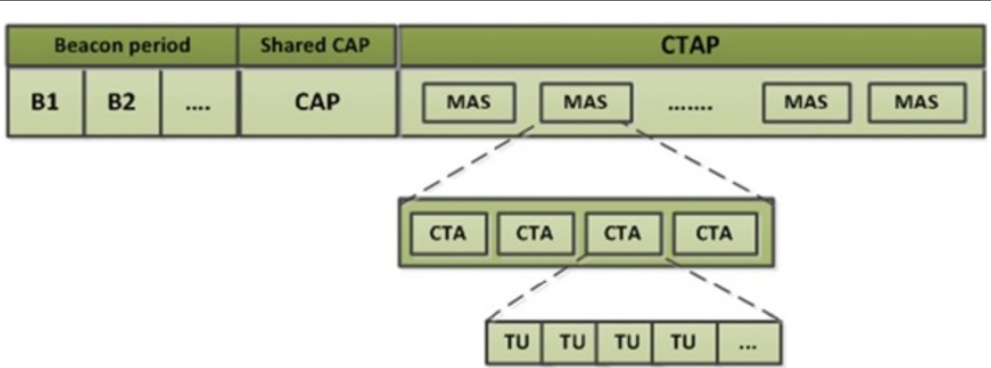

Figure 3 Superframe sharing in IEEE 802.15.5. 


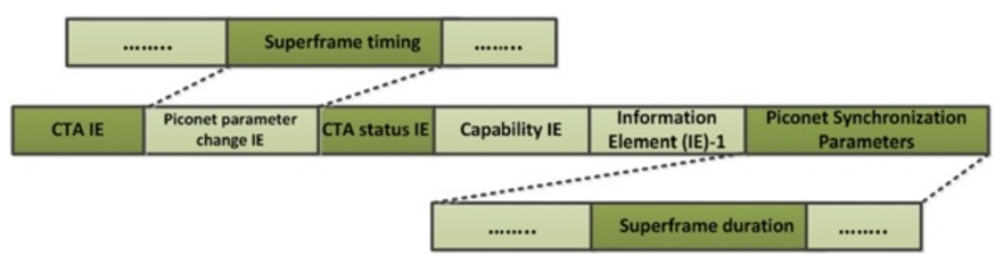

Figure 4 Beacon frame.

and utilization threshold notions. Under the utilization thresholds, two different thresholds are proposed to be used in the resource allocation mechanisms at hop-1, the superframe utilization threshold and the CTAP threshold.

\subsection{Dynamic superframe size}

With this proposal, the notion of dynamic superframe size starting with a minimum value as identified in [1] is introduced. The increase in the superframe size is restricted by the maximum size of the superframe. There exist two different policies that could be used to manage CTAs according to devices' needs. The first is to start off with a maximum superframe length and try to manage the growing number of CTRq commands by proper allocation of CTAs. The second method is to have an initial small superframe length and adjust its length any time it is necessary. The superframe duration can be extracted from the 'superframe duration field' in the 'piconet synchronization parameters' (see Figure 4) that are broadcasted in the beacon.

The size of the superframe is managed by the refMPNC which, based on the novel proposal, recomputes the new superframe size after each CTA request decision. In case of an accepted CTRq, the new allocated channel time size is added to the superframe size and this new size is broadcasted to the devices in the 'superframe timing' field in the piconet parameter change IE (Figure 4) in the beacon. In such cases, the waiting time to start a new superframe is avoided if the maximum superframe size is not reached. Thus, the QoS requirements for real-time traffic in IEEE 802.15.5 can be much satisfied by minimizing the delay between two consecutive CTAs of the same device. Additionally, the superframe capacity is efficiently utilized especially when the network is not overloaded. Consequently, only a portion of the superfame size is used (instead of using its maximum size), thus avoiding the wastage of the superframe capacity.

\subsection{Bulk channel time requests model}

The notion of bulk CTRq (BCTRq) sent by the MPNCs to the ref-MPNC at hop-1 is also considered. In the proposed mechanism, the BCTRqs sent by a MPNC to the ref-MPNC are based on the aggregate of the received CTRqs from its members' MDEVs at hop-2. As highlighted in Figure 5, the MPNCs send BCTRq for requesting CTAs from the ref-MPNC by identifying the 'minimum number of TUs' and the 'desired number of TUs' fields. After receiving the requests, the ref-MPNC can decide to accept or deny the request according to the proposed algorithms, and it notifies the requesting device by sending a CTRp (see Figure 6). The ref-MPNC decision to allocate channel time is based on prioritizing the MPNCs over the MDEVs by granting the MPNCs more TUs.

\subsection{Flag metric notion}

In addition to the use of utilization thresholds, fairness and different priorities are also induced in the proposed mechanism by the use of a priority flag metric (Flag). The ref-MPNC keeps a global record of flag metrics for its members' MDEVs and MPNCs at hop-1. Each hop-1 MPNC also keeps a record of Flag for its member RT and NRT MDEVs at hop-2. Each device is identified at the ref-MPNC by a device identifier (Dev_id) as defined in [9]. Each raised Flag (i.e., its value is greater than zero) refers to a rejected CTRq or BCTRq. The flag metrics list is kept along all the superframe durations. If a device's request is rejected, its flag metric is incremented by one. Otherwise, this metric is decremented, provided that its value is greater than zero (to avoid having a negative flag metric). The flag metrics list is utilized by the ref-MPNC at the CAP of the next superframe to generate a priority list $P$ that includes the flag metrics sorted in a descending order. Then, the ref-MPNC uses $P$ to sort the Dev_id list accordingly, i.e., Dev_id of the device having first metric in $P$ is set at the head of the new Dev_id list. At the CAP of the next superframe, the

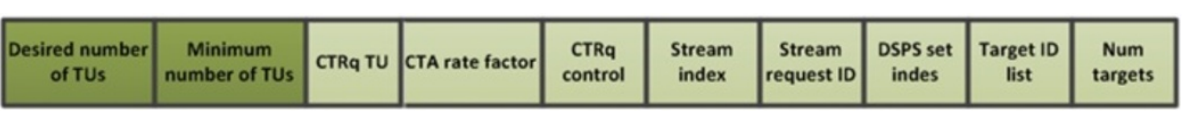

Figure 5 Channel time request block field format. 


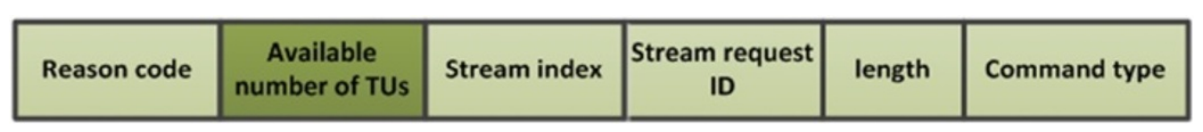

Figure 6 Channel time response command format.

devices' requests are served according to this new Dev_id list. Moreover, the initial flag metrics assigned to the hop1 MPNCs and hop-2 RT MDEVs are higher than that of hop-1 MDEVs and hop-2 NRT MDEVs, respectively.

\subsection{Utilization thresholds}

In order to attract more devices, the network must be able to offer a comparatively satisfactory service to the devices to reduce the loss of devices. However, because of a limited superframe capacity, some devices' requests are rejected in order to satisfy other devices' requirements. Obviously, it is not fair to guarantee the requesting devices' QoS by rejecting more devices. Consequently, a compromise between the devices satisfaction and the requests' rejection rate is applied by the use of utilization thresholds at the hop-1 resource allocation mechanism. These thresholds are the CTAP utilization threshold and the superframe utilization threshold.

\subsubsection{CTAP utilization threshold}

In the proposed mechanism, the CTAP threshold $\left(\mathrm{CTAP}_{-} T_{L}\right)$ is introduced to split the CTAP into two sub-CTAPs, MPNC-CTAP, and MDEV-CTAP. The sizes of these two sub-CTAPS are referred to as CTAP MPNC_size and CTAP_MDEV_size, respectively. These sizes are adjusted according to $\Phi$ as:

$$
\text { CTAP_MPNC_size }=\Phi \times \text { size }_{\max } \text {, where }(0<\Phi<1)
$$

and

$$
\text { CTAP_MDEV_size }=(1-\Phi) \times \text { size }_{\max }
$$

where size $_{\text {max }}$ is the maximum size of the superframe. For providing higher priority for the MPNCs over the MDEVs, a larger CTAP portion is reserved for the
MPNCs, i.e., CTAP_MPNC_size > CTAP_MDEV_size, by adjusting the value of $\Phi$ to be greater than $(1-\Phi)$.

Then, a MPNC threshold (MPNC_ $T_{L}$ ) is introduced in the CTAP_MPNC and is used by the ref-MPNC to adjust the size of the channel time resources to be granted for the requesting MPNCs. Similarly, a MDEV threshold $\left(\mathrm{MDEV}_{-} T_{L}\right)$ is introduced in the CTAP_MDEV which, in its turn, is used by the ref-MPNC to adjust the size of the channel time resources to be granted for the requesting MDEVs (Figure 7). These thresholds are defined as follows:

$$
\text { MPNC_ } T_{L}=\beta 1 \times \text { CTAP_MPNC_size, where }(0<\beta 1<1)
$$

MDEV_T $T_{L}=\beta 2 \times$ CTAP_MDEV_size, where $(0<\beta 2<1)$

where the values of these thresholds are adjusted according to $\beta 1$ and $\beta 2$, respectively. As long as the total allocated channel time size in the CTAP_MPNC and CTAP_MDEV is below the MPNC_ $T_{L}$ and the MDEV $T_{L}$, respectively; the channel times are granted in a way such that the requesting devices are highly satisfied, i.e., the granted channel times are approximately equal to the requested channel times. Otherwise, the ref-MPNC applies a control procedure that minimizes the size of the granted resources in order to allow more devices to get channel access. This is in condition that the granted resources remain greater than or equal to the minimum requested channel times.

\subsubsection{Superframe utilization threshold}

The proposed mechanism applies a common superframe utilization threshold $\left(T_{L}\right)$ for the hop-1 devices (MPNCs and MDEVs) in another hop-1 resource allocation

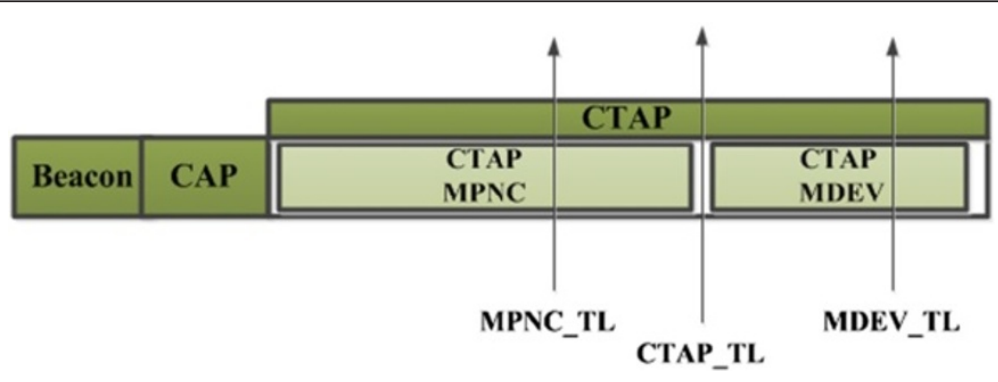

Figure 7 CTAP utilization thresholds. 
algorithm. Then, the prioritization of the MPNCs over MDEVs is applied within this algorithm. The use of $T_{L}$ is such that whenever the total CTAP allocated channel time size is below $T_{L}$, the requesting devices are highly satisfied. Then when the total allocated superframe size exceeds this threshold, the ref-MPNC reduces the granted resource size to allow more devices to get channel access. Nevertheless, it is guaranteed that the granted channels are still greater than or equal to the minimum requested resources by a device. The threshold $T_{L}$ is defined as:

$$
T_{L}=\operatorname{size}_{\max }-2 \times \alpha \times \mathrm{TU} \times \mathrm{N}
$$

where TU is the duration of a time unit in microseconds, $N$ is the total number of devices and $\alpha$ is a factor through which different values of $T_{L}$ can be obtained as illustrated in Figure 8. All the other parameters for calculating $T_{L}$ are considered to be constant in a certain scenario. The reason of the choice of $(2 \times \mathrm{TU} \times N)$ is to ensure that two TUs, as minimum, are available for each of the associated devices to be capable of communicating with the ref-MPNC (one TU for uplink stream and another for downlink stream).

\section{IEEE 802.15.5 proposed resource allocation mechanisms}

The block diagram shown in Figure 9 represents the proposed two-hop IEEE 802.15.5 mechanism which can be decomposed into five stages. It is a two-tier mechanism of hop-1 resource allocation algorithms for the MDEVs and MPNCs and a hop- 2 resource allocation algorithm for the RT and NRT MDEVs. For the hop-1, two different algorithms are proposed at stage (3) and the hop-2 algorithm takes place at stage (5). It is important to mention that the implementation of the hop- 2 algorithm is related to the validity of the condition that the hop- 1 device is a MPNC with granted channel time resources as shown in Figure 9. In the sequel, each block is described in details. These algorithms can be defined as follows:

Hop-1 algorithms: control the admission and resource allocation between the ref-MPNC and its member MDEVs/MPNCs belonging to the parent piconet. Individual CTRqs are sent from MDEVs to ref-MPNC and BCTRqs are sent from MPNCs to ref-MPNC. For the hop-1 mechanism, two algorithms are proposed: the first one is the CTAP utilization threshold-based resource allocation algorithm (CTRA) and the second one is the superframe utilization threshold-based resource allocation algorithm (STRA).

Hop-2 algorithm: controls the admission and resource allocation between the hop-1 MPNCs and their member RT/NRT MDEVs. The concerned MPNC applies the hop-2 algorithm which is a service differentiation-based resource allocation algorithm (SDRA) to distribute the CTAs among the RT and NRT MDEVs.

\section{1 Hop- 1 resource allocation algorithms}

According to the novel proposal, we have two hop-1 resource allocation algorithms, CTRA and STRA. Each of these algorithms can be divided into several stages. These stages are common between both of these algorithms except for the 'hop-1 channel time calculations' stage which is particular for each of these two algorithms. These stages, identified in Figure 9, are described as follows and are detailed accordingly in the form of block diagrams from Figures 10, 11, 12, 13, 14, 15, and 16:

Stage (1) - Initialization and hop-1 Priority list creation: The flow chart corresponding to this stage is described in Figure 10. At the initial stage, the minimum and maximum superframe sizes $\left(\right.$ size $_{\min }$ and size $_{\max }$ ) are set. The ref-MPNC initializes the devices ID list (Dev_id1) of its hop-1 MPNCs and MDEVs. In each superframe $S$, the Flag metric list is generated with initial values for each device. Higher flag metrics are assigned to the MPNCs to give them higher priority than MDEVs. Prior to its channel time requests decision, a ref-MPNC creates a priority list $(P 1)$ from the Flag metric list sorted in a descending order. The Dev_id1 list is then sorted according to $P 1$. The abbreviations' descriptions of all the flowcharts are presented in Table 1.

Stage (2) - Receiving channel time requests: The flowchart of this stage is shown in Figure 11. Whenever the ref-MPNC receives a CTRq/BCTRq, it serves it according to the sorted Dev_id1 list. Since the MPNCs are assigned higher flag metrics at the initialization

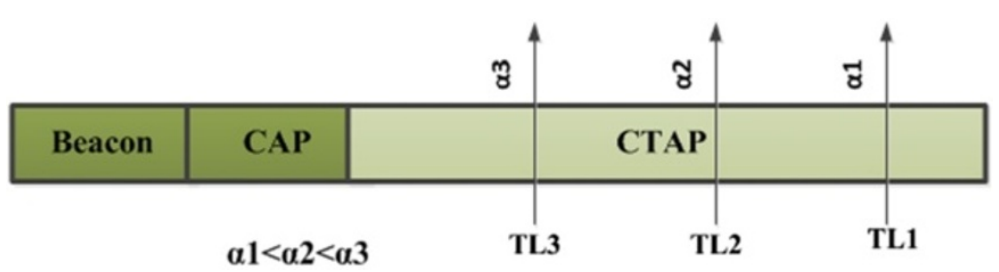

Figure 8 Superframe utilization threshold for different $a$. 

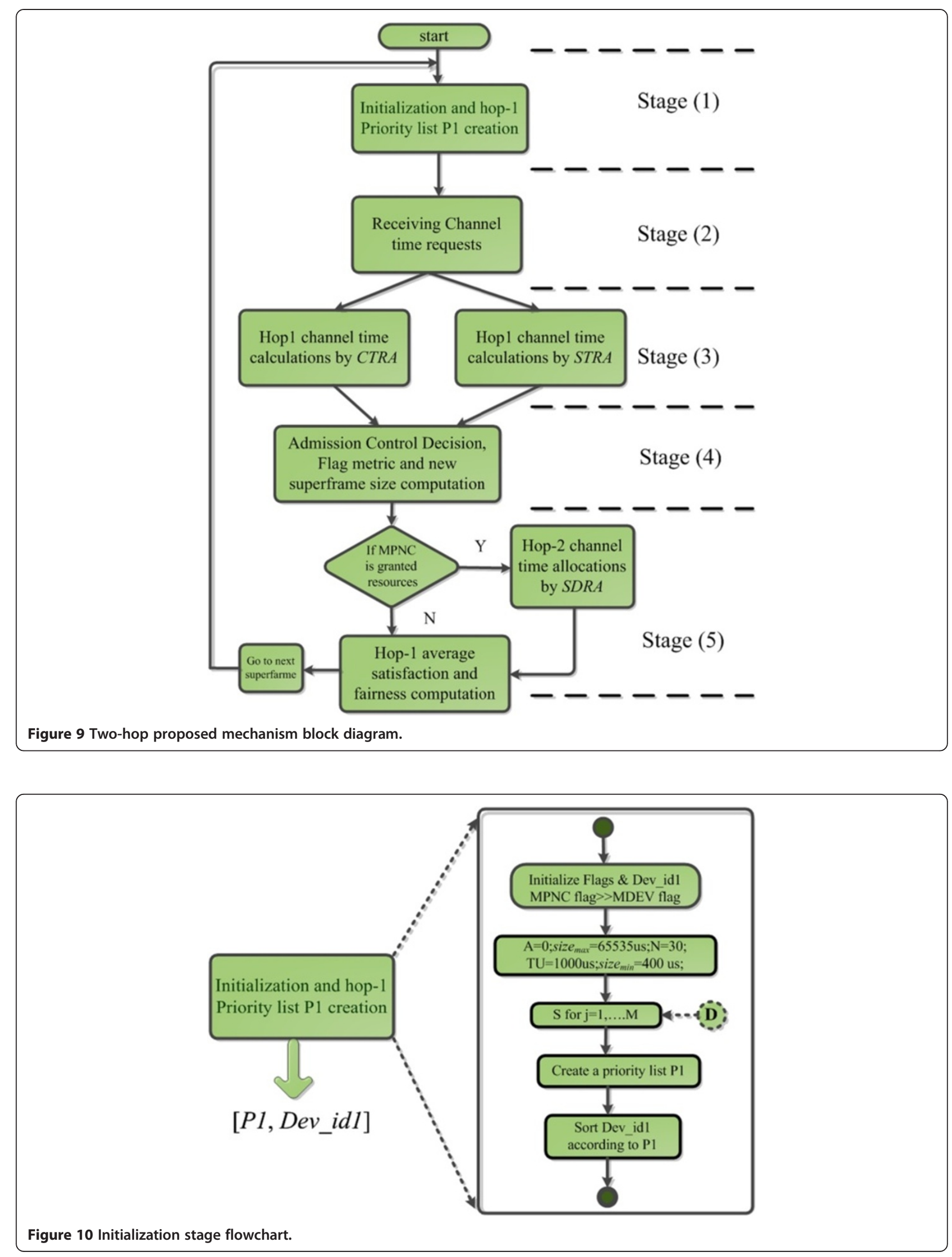


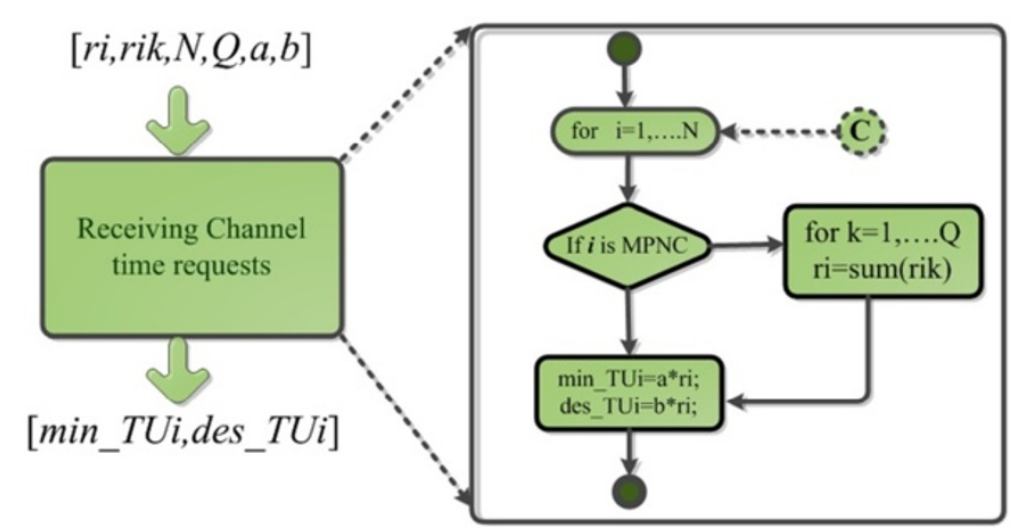

Figure 11 Receiving channel time requests flowchart.

stage, then they occupy the first positions of the Dev_id1 list. The values of the minimum number of TUs $\left(\min \_\mathrm{TU}_{i}\right)$ and the desired number of TUs (des_T $\mathrm{TU}_{i}$ ) of each device $i$ are equal to a fraction of its requested channel time resources $\left(r_{i}\right)$, i.e., $\left(a . r_{i}\right)$ and $\left(b . r_{i}\right)$, respectively, where a and $\mathrm{b}$ values are less than 1 . Stage (3) - Hop-1 channel time calculations: In this stage, the behavior of the two hop-1 algorithms is differentiated as follows:

\subsubsection{CTAP utilization threshold-based resource allocation algorithm}

Firstly, the ref-MPNC checks if the request received is sent by a MPNC or a MDEV (see Figure 12). Consequently, it checks the MPNC_ $T_{L}$ or MDEV_T $T_{L}$, and it then grants the channel times accordingly. Additionally, the ref-MPNC has to grant channel times for the MPNCs from the CTAP_MPNC portion in the shared superframe according to the MPNC_ $T_{L}$. As long as the total allocated CTAP_MPNC channel time $\left(A_{\text {mpnc }}\right)$ does not exceed MPNC_ $T_{L}$, the ref-MPNC grants up to the des_TU $\mathrm{U}_{i}$ for a BCTRq. Otherwise, it indicates to the attached devices in the capability IE in the beacon (Figure 4) about the new capacity constraints and it reduces the size of the granted channel times to the average of the min_ $\mathrm{TU}_{i}$ and des_ $\mathrm{TU}_{i}$ for the requesting MPNC.

Similarly for the MDEV CTRqs, when the total allocated CTAP_MDEV channel time $\left(A_{\text {mdev }}\right)$ is below MDEV_ $T_{L}$, the MDEVs are granted up to the average of the min_TU $U_{i}$ and des_TU $\mathrm{T}_{i}$. When the total MDEVs' allocated resources exceed MDEV_T $T_{L}$, the ref-MPNC indicates to the attached devices in the capability IE about the new capacity constraints. From now on, the refMPNC accepts requests with new constraints by granting up to the min_TU $\mathrm{TU}_{i}$ for the requesting MDEVs. In the proposed algorithms, the calculated channel times are referred to as $r q_{i}$, then if these resources are granted successfully we refer to them as $g_{i}$.

\subsubsection{Superframe utilization threshold-based resource allocation algorithm}

The algorithm flowchart of the proposed mechanism is shown in Figure 13. The ref-MPNC grants up to the des_TU $U_{i}$ for a BCTRq, while it grants up to the average

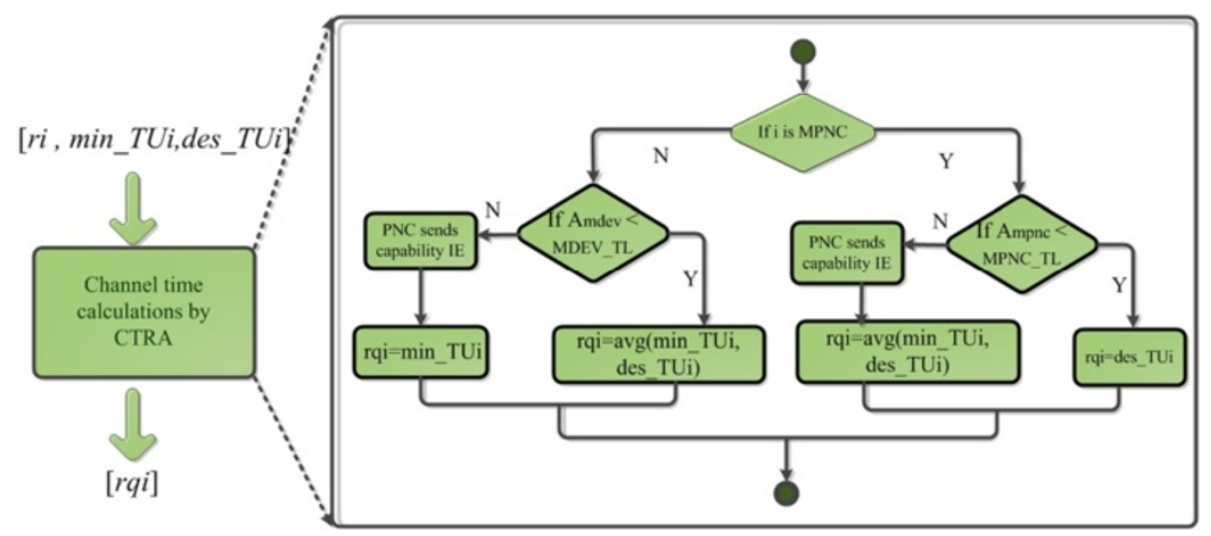

Figure 12 CTRA resource allocation algorithm. 


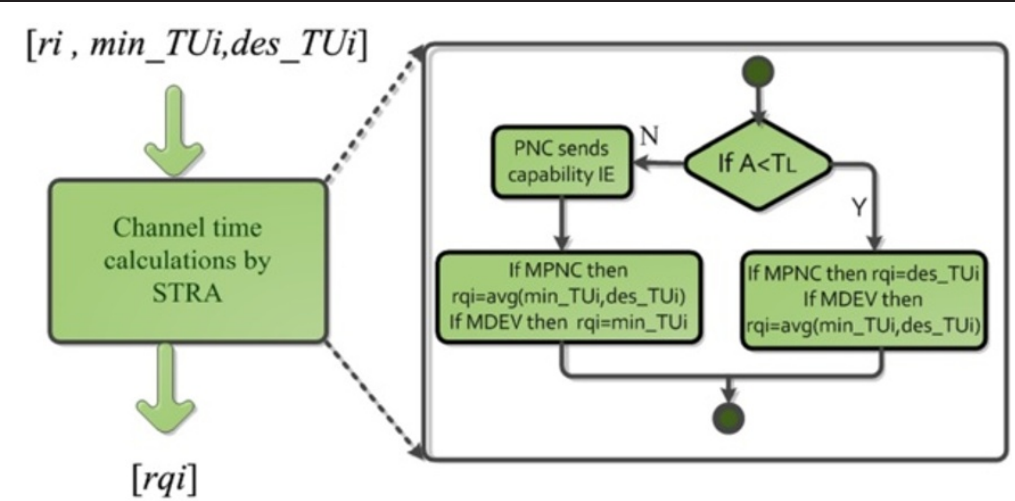

Figure 13 STRA resource allocation algorithm.

of the min_ $\mathrm{TU}_{i}$ and des_TU $\mathrm{U}_{i}$ for a CTRq. This is applied as long as the total allocated channel time $(A)$ is below a certain superframe utilization threshold $T_{L}$. When the allocated resources exceed $T_{L}$, the ref-MPNC indicates to the attached devices in the capability IE (in the beacon) about the new capacity constraints the ref-MPNC is capable of handling. Later on, the ref-MPNC accepts requests with new constraints, it grants up to the average of the min_TU $\mathrm{TU}_{i}$ and des_TU $\mathrm{TU}_{i}$ for MPNCs and up to the min_TU $\mathrm{TU}_{i}$ for the MDEVs.

Stage (4) - Admission control decision: the flowchart of this stage is shown in Figure 14. For both hop-1 algorithms, the conditions applied on the size of channel time to be granted $\left(r q_{i}\right)$ are applied as long as the summation of $(A)$ and $\left(r q_{i}\right)$ does not exceed the superframe maximum capacity $\left(\right.$ size $\left._{\max }\right)$; otherwise, the requests are rejected. In the case where a BCTRq/CTRq is rejected, the Flag metric of the concerned MPNC/MDEV is incremented and a negative CTRp is sent by the refMPNC. Otherwise, a positive CTRp is received by the device with the granted channel time resources $\left(g_{i}\right)$ and its Flag metric is decremented by one. In both CTRA and STRA, a satisfaction factor of each device $\left(\right.$ sat $\left._{f i}\right)$ is calculated as defined in Equation 7. The new granted resources amount $\left(g_{i}\right)$ is added to the total allocated resources $(A)$ during a superframe. The new superframe size $\left(S_{\mathrm{z}}\right)$ is then calculated by the addition of $(A)$ to size $\mathrm{min}_{\mathrm{min}}$ as follows:

$$
\mathrm{S}_{\mathrm{z}}=\text { size }_{\min }+\mathrm{A}
$$

The satisfaction factor of a device $i$ is defined to be equal to the ratio of granted TUs $\left(g_{i}\right)$ divided by the desired TUs $\left(\right.$ des_TU $\left._{i}\right)$ requested by a device $i$ as:

$$
\operatorname{sat}_{f_{i}}=\frac{g_{i}}{{\operatorname{des} \_U_{i}}_{\mathrm{TU}_{i}}}
$$

Stage (5) - Average satisfaction and fairness computation: Before moving to the next superframe $(j+1)$, the hop-2 resource allocation algorithm is applied to the hop- 2 members only if the hop- 1 member is a MPNC and it has been granted channel time resources, i.e., $g_{i}>0$ (Figure 15). Then the algorithm returns back to the point number (C) identified in Figure 11 to repeat stages (2) and

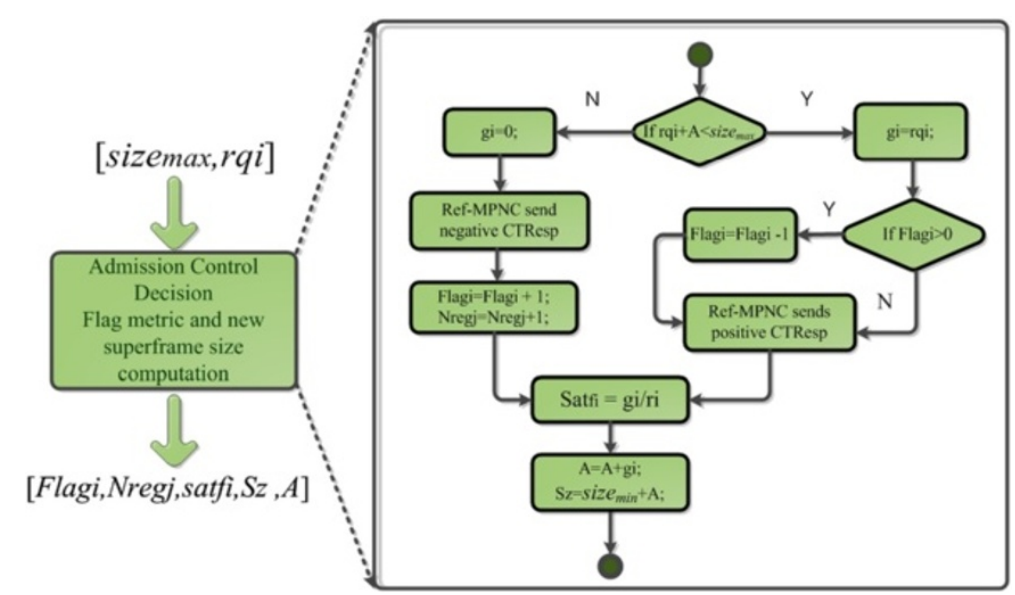

Figure 14 Admission control and satisfaction stage. 


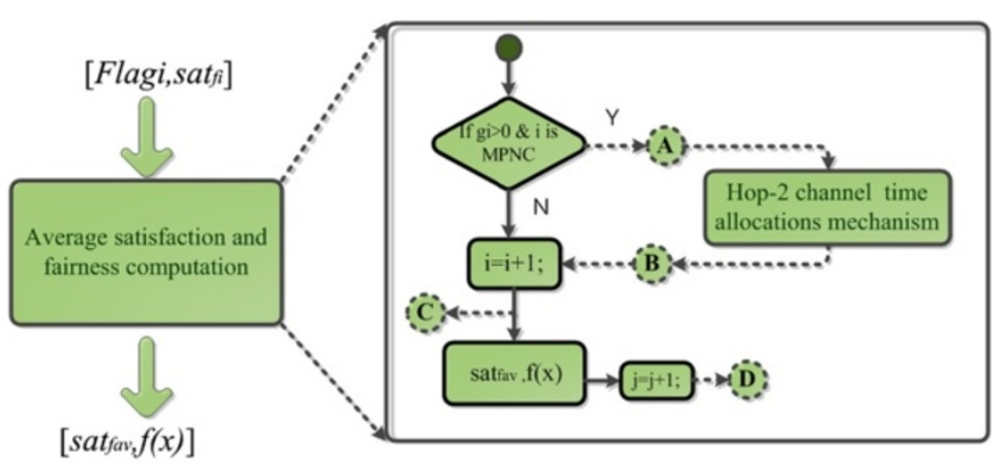

Figure 15 Average satisfaction and fairness computation stage flowchart.

(3) for all the other hop-1 devices until $i$ reaches $N$. Next, it calculates the average satisfaction factor of all the devices after calculating the device satisfaction factor at stage (3). The average satisfaction factor is defined as:

$$
\operatorname{sat}_{f_{\mathrm{av}}}=\frac{\sum_{i} \mathrm{sat}_{f i}}{n}
$$

where $n$ represents the number of devices (MPNCs or MDEVs). Using only sat $f_{\text {av }}$ value is not enough to determine the fairness of the proposed resource allocation mechanism. It is highly desirable to find a method and, preferably, an index to measure and compare the degree of fairness of a particular allocation policy [15]. Many general purpose fairness indexes (Gini, Jain's Fairness, min-max) have been frequently used to measure fairness of different resource allocation schemes $[16,17]$. We apply the Jain's Fairness index on the satisfaction factor which is in function of granted resources and requested resources as defined in Equation 7. The Jain's Fairness index is defined as follows:

$$
f(x)=\frac{\left(\sum_{i} \mathrm{sat}_{f_{i}}\right)^{2}}{N \sum_{i} \mathrm{sat}_{f i}{ }^{2}} \forall i=1,2, . . N
$$

where $f\left(x=\operatorname{sat}_{f}\right)$ is the Jain's Fairness index and $N$ is the total number of devices. The fairness index value is a positive number with max value 1 indicating a $100 \%$ fair system.

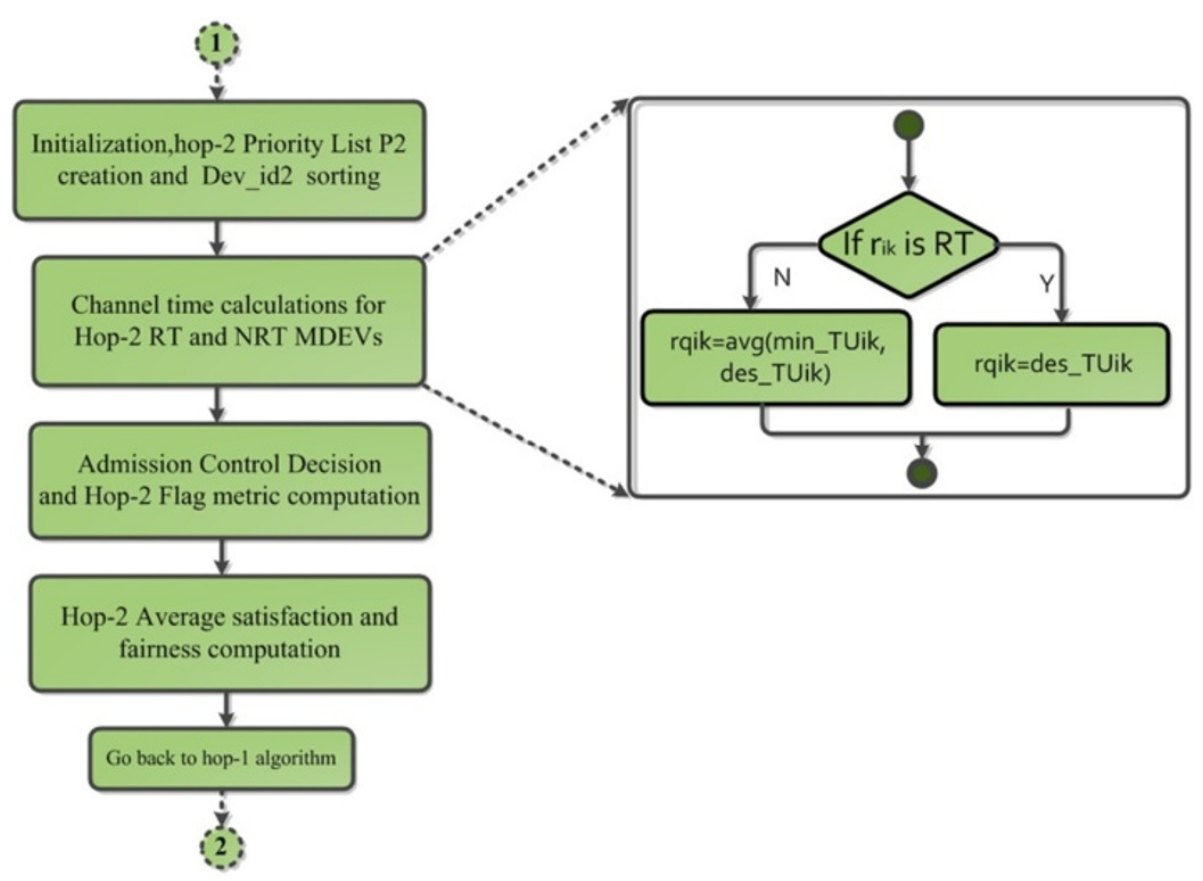

Figure 16 SDRA resource allocation algorithm. 
Table 1 Flowcharts abbreviations

\begin{tabular}{|c|c|}
\hline Abbreviation & Description \\
\hline Dev_id1, Dev_id2 & $\begin{array}{l}\text { Array of device ID for the devices at hops } 1 \\
\text { and } 2 \text {, respectively }\end{array}$ \\
\hline$P 1, P 2$ & Priority lists at hops 1 and 2, respectively \\
\hline A & Total allocated channel time of the superframe \\
\hline$A_{\mathrm{mpnc}} A_{\mathrm{mdev}}$ & $\begin{array}{l}\text { Total allocated channel time by hop-1 MPNCs } \\
\text { and MDEVs, respectively }\end{array}$ \\
\hline S & Number of superframes \\
\hline$S_{z}$, size $_{\max }$, size $_{\min }$ & $\begin{array}{l}\text { Current, maximum and minimum sizes of } \\
\text { superframe }(\mu s)\end{array}$ \\
\hline$r_{i r} r_{i k}$ & $\begin{array}{l}\text { Requested channel time by a device at hops } 1 \\
\text { and } 2 \text {, respectively }\end{array}$ \\
\hline$r q_{i}, r q_{i k}$ & $\begin{array}{l}\text { Size of channel time size to be granted to a } \\
\text { device if available at hops } 1 \text { and } 2 \text {, respectively }\end{array}$ \\
\hline$g_{i}$ & $\begin{array}{l}\text { Granted channel time to device according to } \\
\text { different algorithms }\end{array}$ \\
\hline$N, Q$ & Number of devices at hops 1 and 2, respectively \\
\hline$M P N C_{-} T_{L}, M D E V_{-} T_{L}$ & $\begin{array}{l}\text { MPNC and MDEV CTAP utilization threshold, } \\
\text { respectively }\end{array}$ \\
\hline$N_{\text {regj }}$ & Number of rejected requests in a superframe $j$ \\
\hline TU & Duration of a time unit $(\mu s)$ \\
\hline $\min \_T U_{i}$, des_TU ${ }_{i}$ & $\begin{array}{l}\text { Minimum and desired number of TUs requested } \\
\text { by a device } i\end{array}$ \\
\hline$a, b$ & $\begin{array}{l}\text { Ratio of min_TU and des_TU, respectively, } \\
\text { with respect to request }\end{array}$ \\
\hline sat $_{f i}$ sat $_{f a v}$ & $\begin{array}{l}\text { Calculated satisfaction factor for a device } i \text { and } \\
\text { average satisfaction for } n \text { devices }\end{array}$ \\
\hline$f(x)$ & Jain's Fairness index \\
\hline
\end{tabular}

After that, the algorithm proceeds to the next superframe by returning back to point (D) identified in Figure 10.

\subsection{Hop-2 service differentiation-based resource allocation algorithm}

The most relevant work for resource allocation for hop1 WPANs mesh is given in [12] as previously mentioned. In addition to the already identified drawbacks, the work in [12] does not present a mechanism for distributing the MPNCs' resources among their piconets' member MDEVs. In this scheme, a hop-2 centralized service differentiation-based resource allocation algorithm (SDRA) in IEEE 802.15.5 is proposed using the approaches that are applied by the hop-1 algorithms. Whatever the applied hop-1 algorithm (CTRA or STRA) is, the hop-2 algorithm (SDRA) does not change. This algorithm is proposed for fairly sharing the hop-1 MPNC resources among its hop-2 RT and NRT MDEVs. Additionally, this algorithm prioritizes RT over NRT MDEVs and grants them larger CTA allocations. SDRA, which is started at the point number (A) in Figure 15, can be divided into the same stages described for the hop-1 algorithms as illustrated in Figure 16 but with the following differences:

1) In SDRA, all the stages described for the hop-1 algorithms are performed by the hop-1 MPNC instead of the ref-MPNC.

2) At the initialization stage, initial higher priority is given to the RT MDEVs over the NRT MDEVs by assigning the former higher Flag metrics than the latter.

3) Different rules are applied at the 'channel time calculations' stage where a MPNC $i$ has to distribute the channel time among its MDEVs according to their requested resources with service differentiation. The MPNC checks if the CTRq of a device $k$ is for RT or NRT MDEV. Accordingly, it then sets the size of resources to be granted for an RT CTRq $\left(r q_{i k}\right)$ to des_TU $i k$, and to the average of min_TU $U_{i k}$ and des_TU $U_{i k}$ for an NRT CTRq.

4) At the admission control stage, the sum of the total allocated resources is compared with the time granted to a MPNC $i$ ( $\left.g_{\text {impnc }}\right)$ instead of the maximum superframe size.

5) After computing the average satisfaction factor and fairness for the hop-2 RT and NRT MDEVs at the 'average satisfaction and fairness computation' stage, the algorithm returns back to the applied hop-1 algorithm at point (B) identified in Figure 15. It is necessary to highlight the point that the QoS requirements (for example delay) for the RT CTRqs are included in the demanded des_TU, thus in the calculated RT satisfaction factor.

In the proposed scenarios, when applying the CTRA as the hop-1 algorithm with SDRA at hop-2, the twohop mechanism is referred to as CTAP utilization threshold-service differentiation-based mechanism (CTSD). Similarly, when applying the STRA as the hop-1 algorithm with SDRA at hop-2, the two-hop mechanism is referred to as superframe utilization threshold-service differentiationbased mechanism (STSD).

\section{Simulations and results}

The proposed algorithms aim to identify the number of TUs to be allocated to each device as described above. To study the performance of these algorithms and without loss of generality, a two-hop meshed scenario with a ref-MPNC having $20 \mathrm{MDEVs}$ and $10 \mathrm{MPNCs}$ as hop-1 members is considered. Then each MPNC embeds 2 RT and 3 NRT MDEVs as its piconet members as illustrated in Figure 17. Therefore, we have a total of 30 devices at hop-1 and 50 devices at hop-2 distributed among 10 hop2 piconets. This can be considered as a large number of 


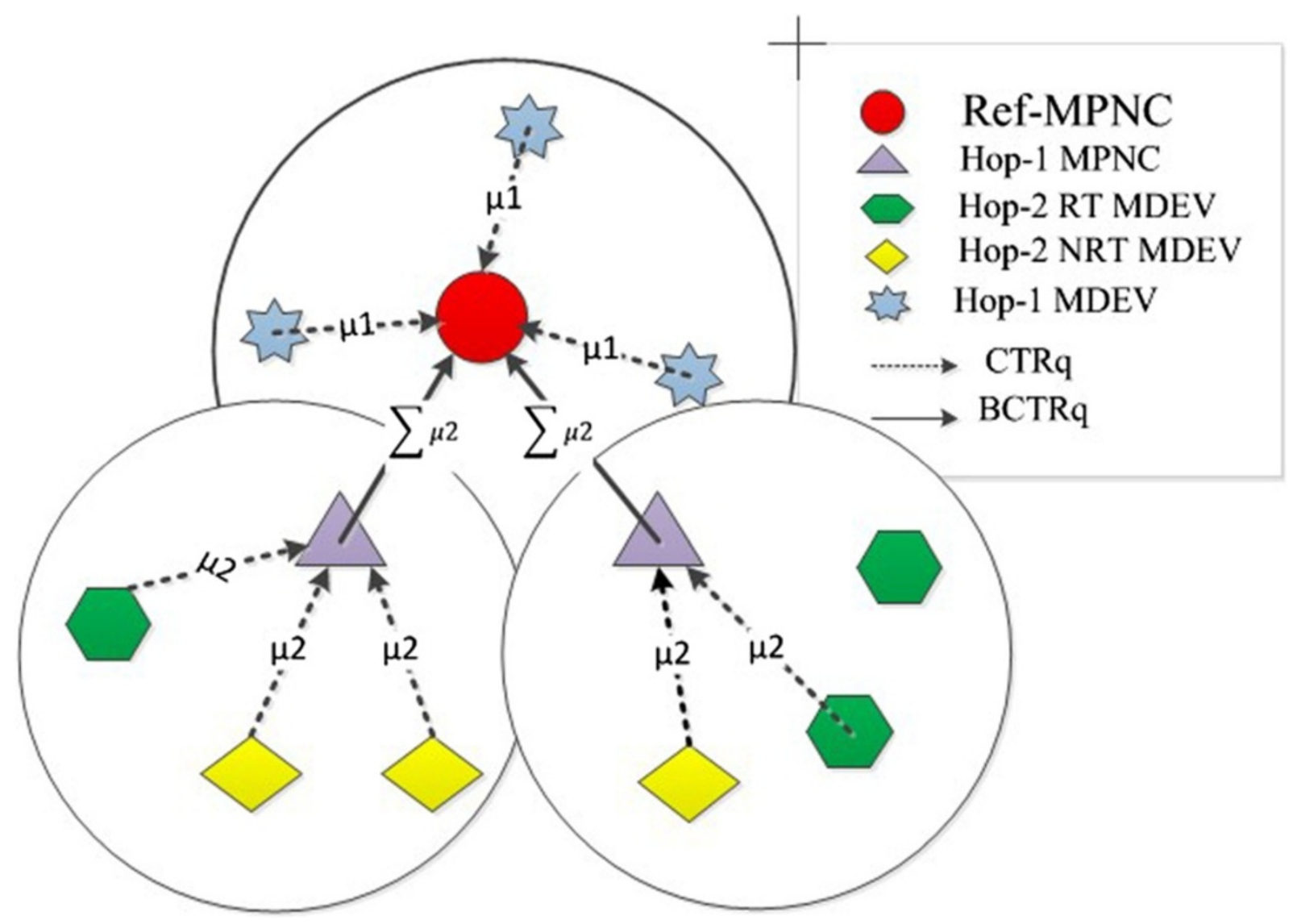

Figure 17 Two-hop IEEE 802.15.5 simulated scenario.

devices in a two-hop WPAN whose piconets do not exceed a 10-m coverage range.

The requests sent by the hop-2 MDEVs to their MPNCs are considered to follow an exponential distribution with mean equals to $(\mu 2)$ microseconds. The $(\mu 2)$ refers to the mean of the individual requests sent by the hop-2 MDEVs to their hop-1 MPNCs. Then, the aggregate of these requests $(\Sigma \mu 2)$ is sent by the hop-1 MPNCs to the ref-MPNC (Figure 17). Additionally, the hop-1 MDEVs send individual requests to the ref-MPNC, which follow an exponential distribution with mean equals to $(\mu 1) \mu$ s. One request per each device is sent during a CAP. Several MATLAB simulations were run by using the parameters' values that are summarized in Table 2, while taking different values of the means $(\mu 1)$ and $(\mu 2)$ as specified in the sequel. The satisfaction factor, the average requests' rejection rate, the average superframe utilization and the fairness index variations are studied.

Figure 18 shows a comparison of the superframe utilization for different loads $(\mu 1)$ when using the dynamic superframe size proposal or fixing the superframe size to its maximum value. It is shown that in case of the proposed mechanism, the superframe is efficiently utilized $(100 \%)$ since its size increases regarding the network load. However, in case of the fixed superframe size, where the maximum size is always used, the superframe is inefficiently utilized in cases of low- and mediumloaded networks. This consequently imposes more delay between two CTAs for a certain device in two subsequent superframes especially for real-time application devices.

\section{Table 2 Simulation parameters}

\begin{tabular}{lc}
\hline Parameter & Value \\
\hline TU duration & $1,000 \mu \mathrm{s}$ \\
min_TU & $0.4 r$ \\
des_TU & $0.9 r$ \\
Number of MPNCs at hop 1 & 10 \\
Number of MDEVs at hop 1 & 20 \\
Number of RT MDEVs at hop 2 per 1 MPNC & 2 \\
Number of NRT MDEVs at hop 2 per 1 MPNC & 3 \\
D, $\beta 1, \beta 2$ & $0.6,0.7,0.7$ \\
Number of superframes S & $10^{5}$ \\
\hline
\end{tabular}




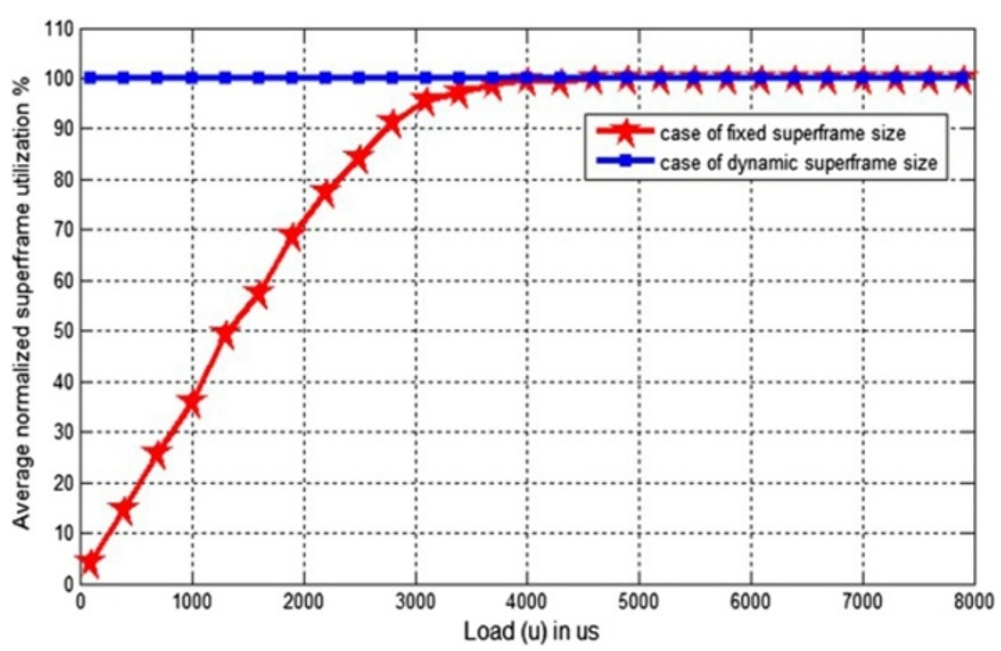

Figure 18 Comparison of average superframe utilization between dynamic and fixed superframe size approaches for different $\mu 1$.

Figure 19 and Figure 20 show the average satisfaction factor of MPNCs/MDEVs and RT/NRT MDEVs, respectively, when applying each of the hop-1 algorithms (CTRA and STRA) in combination with SDRA at hop-2, thus leading to the already defined CTSD and STSD approaches. Therefore, the results presented in the sequel show the performance of the whole two-hop simulated scenario for each of the hop-1 and hop-2. The simulation time is equal to $10^{5}$ superframe durations, and constant values of $\mu 1$ and $\mu 2$ for both hops are considered. It is shown that the MPNCs satisfaction (Figure 19) and the RT MDEVs satisfaction (Figure 20) are very high, and reach 0.9 when applying STSD. This refers to the maximum satisfaction since the des_TU is set to $(0.9 r)$. Consequently, if the des_TU has been set to $r$, the satisfaction would have reached 1 .

When applying STSD, the CTAP is shared among the MPNCs and the MDEVs with one common threshold $T_{L}$. Additionally, the MPNCs are assigned higher priorities than the MDEVs, thus the MPNCs dominate the shared CTAP channel times and reserve their required resources at hop-1. Then, the remaining CTAP channel

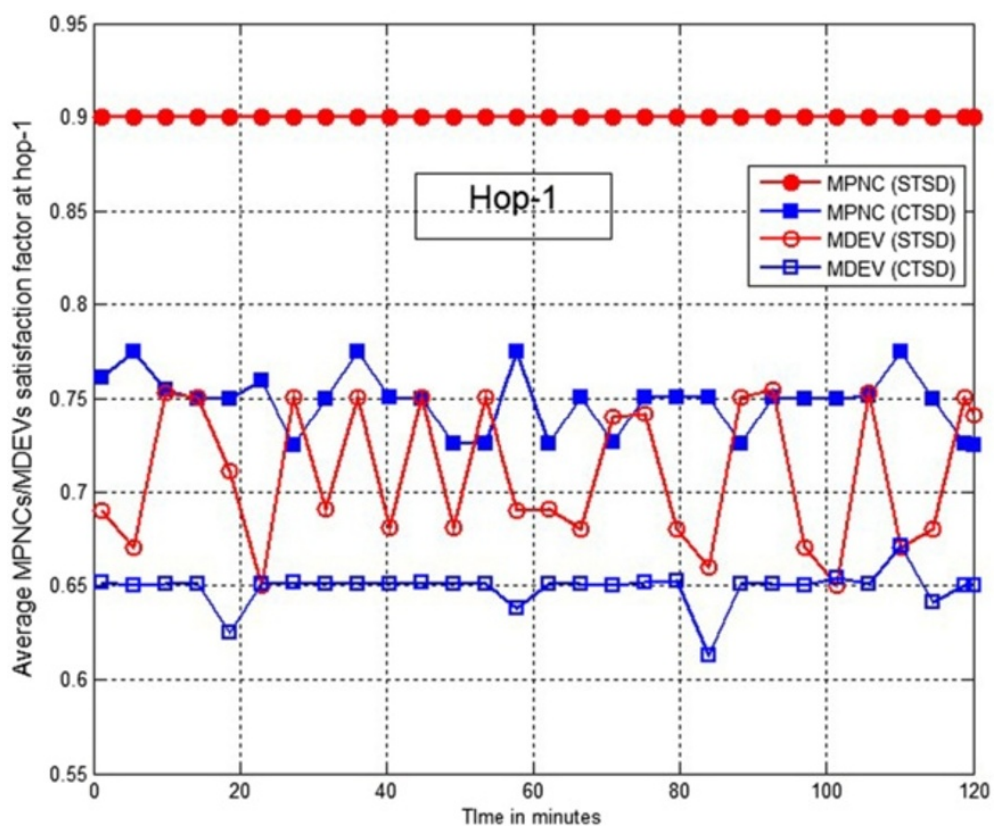

Figure 19 Average MPNCs/MDEVs satisfaction factor at hop- 1 for fixed $\mu 1$ and $\mu 2$. 


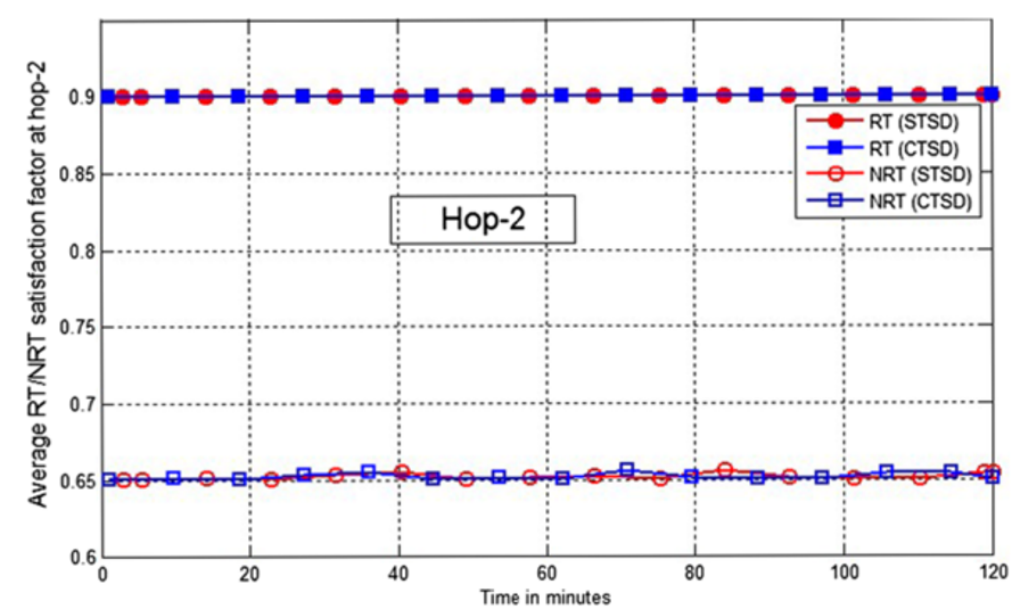

Figure 20 Average RT/NRT MDEVs satisfaction factor at hop-2 for fixed $\mu 1$ and $\mu 2$.

times are distributed among the MDEVs according to their Flag metrics. This explains the much higher MPNCs satisfaction factor compared to the MDEVs satisfaction as illustrated in Figure 19. On the other hand, each of the MPNCs and MDEVs is reserved a CTAP portion when applying CTSD, thus the MPNCs do not grip the MDEVs' channel times. This explains the smaller difference between MPNCs and MDEVs satisfaction in comparison with that using STSD. However, the MPNCs CTAP portion and the MPNCs priorities are higher than those of MDEVs, thus the MPNCs satisfaction is greater than that of MDEVs.

Nevertheless, the same satisfaction factor is achieved by the RT and NRT MDEVs at hop-2 using either STSD or CTSD (see Figure 20) for fixed load. This is because the hop-2 algorithm grants the resources to the hop-2 devices in the same manner regardless of the applied hop-1 algorithm. Moreover, the hop-2 algorithm is applied only in case the hop-1 MPNC is granted its requested resources which are the sum of its hop-2 MDEVs requests; therefore, these MDEVs are granted all their requested resources. Additionally, since RT MDEVs are assigned higher priorities, their satisfaction factor is much greater than that of the NRT MDEVs. Finally, compared to the results obtained by applying CTSD, STSD provides much better results for MPNCs and MDEVs at hop-1 but the RT and NRT satisfaction at hop- 2 remains the same regardless of the applied hop- 1 algorithm.

In the following simulations, the impact of varying the load of both hops on the satisfaction factor, the rejection rate and the Jain's Fairness index, is studied. We take different hop-1 load values $(\mu 1)$ ranging from low-, medium-, to high-loaded networks, and three values of load $\mu 2(200,1,000$, and 2,000 $\mu \mathrm{s})$ that refer to low-, medium-, and high-loaded hop-2 networks, respectively.
Figure 21 and Figure 22 show the variations of the average satisfaction factor of MPNCs and MDEVs when the load at hop-1 increases. Various curves are given for both STSD and CTSD algorithms and for different loads at hop-2. It can be shown that for both algorithms, the MPNCs satisfaction is always higher than that of MDEVs. It is also shown that applying the STSD provides higher satisfaction for both MPNCs and MDEVs for different loaded hop-1 and hop-2 networks. Figure 21 also shows that the MPNCs satisfaction is maintained at 0.9 when applying STSD, whereas it decreases with the increase of the load at both hops when applying CTSD. This is because the CTAP channel times are shared among the MPNCs and MDEVs when applying STSD. Since higher priority is given to the MPNCs, then they usually get all their requested channel times. While for the MDEVs, their satisfaction decreases as the load on both hops decreases as shown in Figure 22. However, in the case of applying CTSD, the CTAP is divided into two parts, one is reserved for the MPNCs and the other for the MDEVs. So even though the MPNCs are provided with higher priority than the MDEVs, they cannot use the resources reserved for the MDEVs. Thus, as the load increases, the same behavior is shown for both MPNCs and MDEVs where the possibility of rejecting or granting smaller channel times than the requested by MPNCs or MDEVs increases. This explains the decrease in the MPNCs and MDEVs satisfaction and the increase in the rejection rate as the load increases as shown in Figure 23. However, the MPNCs satisfaction is still much higher than that of the MDEVs because the MPNCs reserved CTAP portion is larger than that of MDEVs.

Comparing the RT and NRT results at hop-2, it can be shown in Figure 24 and Figure 25 that the RT satisfaction is higher than that of the NRT. In addition, it is 


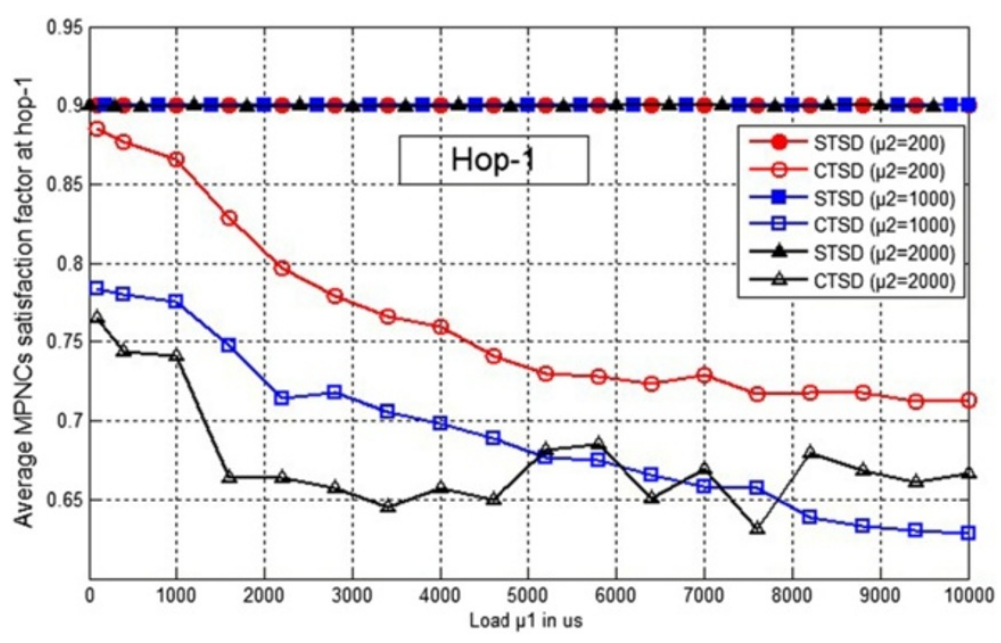

Figure 21 MPNC satisfaction at hop- 1 for different $\mu 1$ and $\mu 2=200,1,000,2,000 \mu \mathrm{s}$.

noticed that the RT satisfaction remains the same regardless of the applied hop-1 algorithm and for different loads at both hops. This is because the RT requests are provided with higher priority than the NRT requests, so they get their requested channel times (this is why satisfaction is 0.9) and larger CTA sizes. It is also shown in Figure 25 that applying STSD at hop-1 gives better satisfaction for the NRT than applying the CTSD because the NRT satisfaction depends on the size of available hop-1 MPNC channel resources. Since STSD provides larger CTA allocations for the MPNCs than the CTSD, then the capacity of the hop-1 MPNCs is larger when applying the STSD, which finally translates into better results at hop-2.

In Figure 26, it is shown that the average rejected request rate is negligible at hop-2 when applying any of the hop-1 algorithms for different loaded networks. This is because the hop-2 algorithm is implemented only if the hop-1 MPNC is granted its requested channel times. This guarantees that the hop-2 devices are approximately granted their desired channel time size. However, a minor request rejection rate is noticed at high-loaded hop-2 networks $(\mu 2=2,000 \mu \mathrm{s})$ when applying CTSD since the latter achieves the lowest MPNC satisfaction factor (i.e., the granted resource size) for $\mu 2=2,000 \mu \mathrm{s}$ as already shown in Figure 21.

For the Jain's Fairness index shown in Figure 27 and Figure 28, it is obvious that both algorithms provide an approximate of $100 \%$ fairness for hop- 2 devices for different hop-1 loaded networks and for both algorithms (Figure 28). This is because of the same reason explained in the previous paragraph which states that the hop- 2

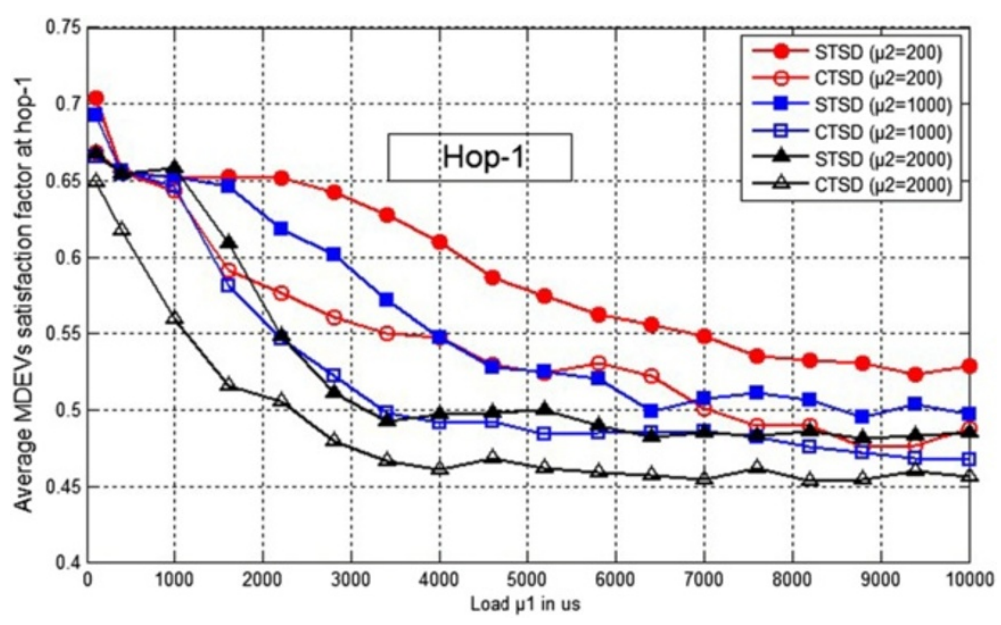

Figure 22 MDEV satisfaction at hop- 1 for different $\mu 1$ and $\mu 2=200,1,000,2,000 \mu s$. 


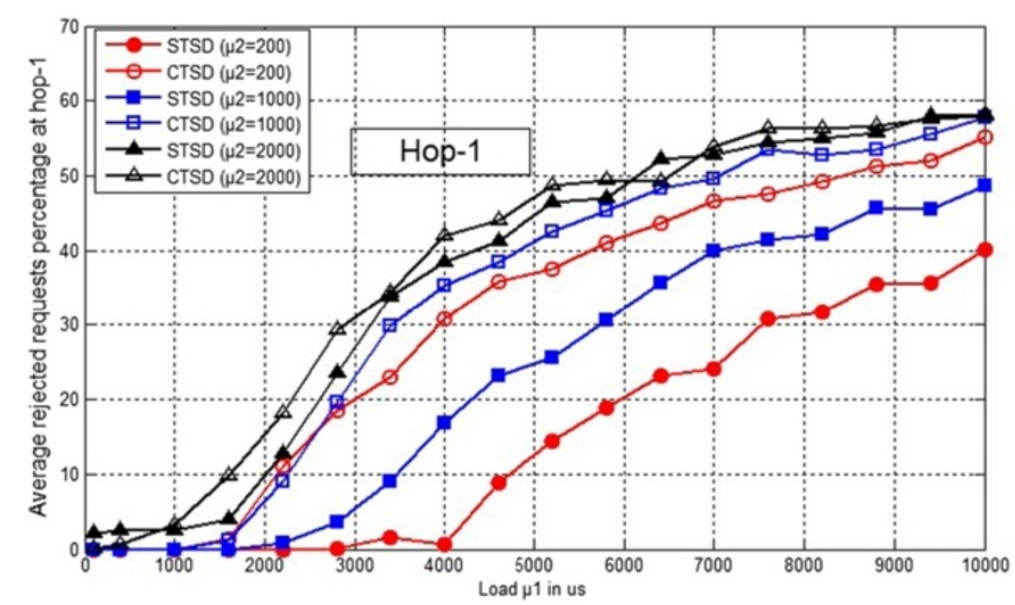

Figure 23 Average rejected requests percentage at hop- 1 for different $\mu 1$ and $\mu 2=200,1,000,2,000 \mu$ s.

devices are granted their desired channel time size. However, the fairness at hop-1 is very high at low-loaded hop-1 and hop- 2 networks and starts to decrease as load at both hops increases (Figure 27). It is also noticed that better fairness is obtained when applying the STSD compared to applying CTSD. This is because higher MPNCs and MDEVs satisfaction rate and lower rejection rate are obtained when applying the STSD which implement the $T_{L}$ notion. Thus, using $T_{L}$ makes the system more adaptive inducing a compromise between the satisfaction and the rejection rate of the devices (as explained previously).

Concerning the hop-2 simulation results shown above, it is worthy to mention that studying only the performance of SDRA independently of the hop-1 algorithms cannot be implemented in this work. This is because the implementation of SDRA is dependent on the validity of the condition that the hop-1 MPNC is granted resources by the running hop-1 algorithm as mentioned previously.
After showing in Figure 18 that the superframe dynamicity enhances the superframe utilization and the delay, we try to show the usefulness of the use of the Flag metric and the utilization thresholds in this part. Therefore, a comparison of the MPNCs/MDEVs satisfaction and fairness indexes between the proposed mechanism that jointly applies the utilization thresholds and priority list and without applying this mechanism is conducted. Since it is concluded from the previous analyses that STSD (i.e., STRA at hop-1) outperforms CTSD (i.e., CTRA at hop-1), only STRA is applied in the sequel. Therefore, comparing the resultant satisfaction factor and the average Jain's Fairness of the proposed mechanism with and without STRA is directly reflecting and showing the usefulness of applying the Flag metric and the superframe utilization threshold which are the basis of STRA.

Figure 29 shows a comparison of the MPNCs and the MDEVs average satisfaction factor for different loads $\mu 1$

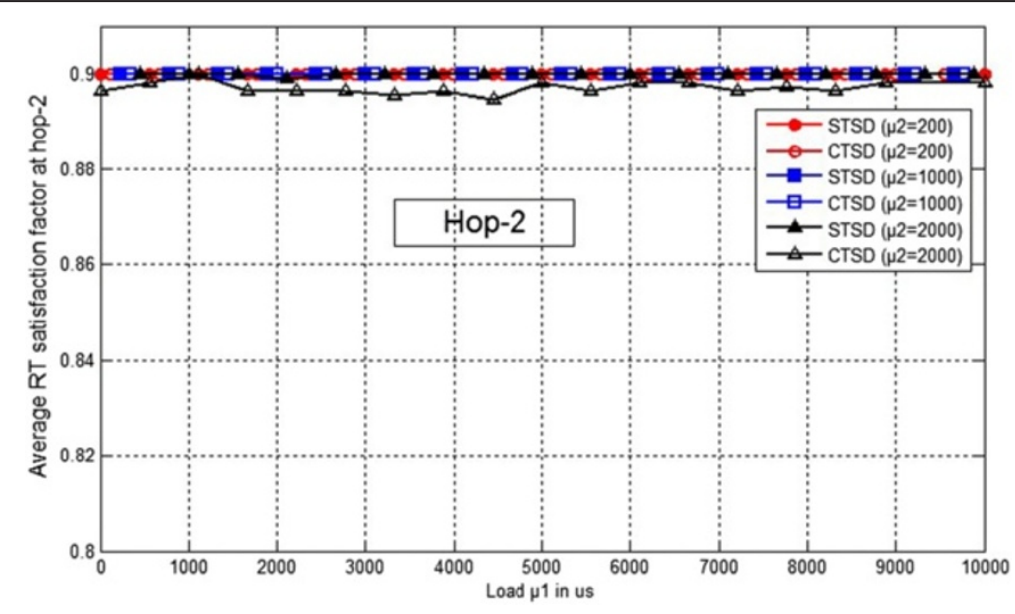

Figure 24 RT MDEV average satisfaction at hop-2 for different $\mu 1$ and $\mu 2=200,1,000,2,000 \mu \mathrm{s}$. 


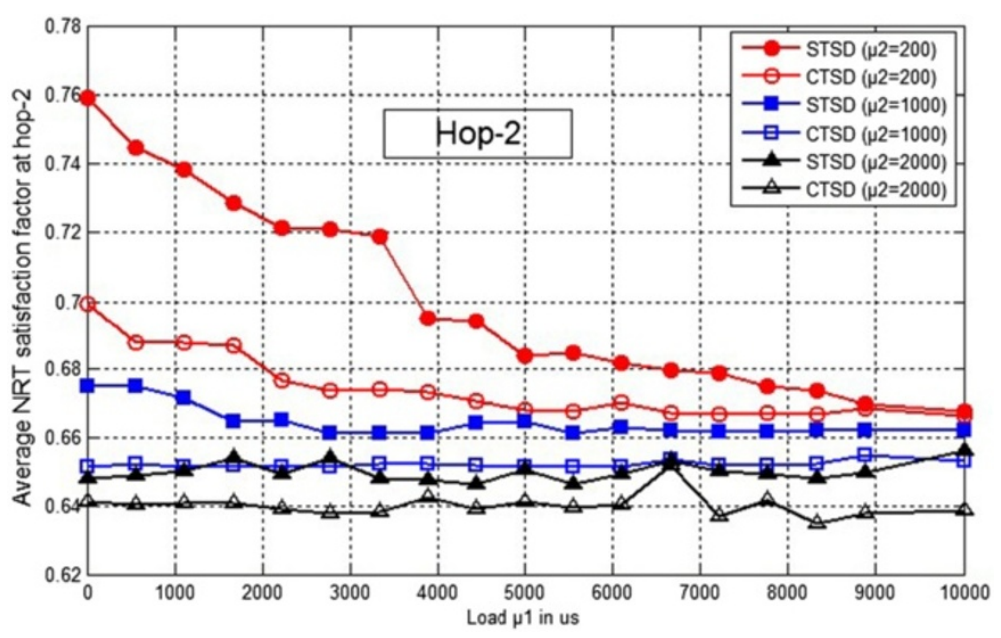

Figure 25 NRT MDEV average satisfaction at hop-2 for different $\mu 1$ and $\mu 2=200,1,000,2,000 \mu \mathrm{s}$.

with or without applying STRA. It is shown that much higher satisfaction is obtained thanks to the STRA for both MDEVs and MPNCs. Moreover, the variations obtained in the case of the STRA reveal the use of the threshold $T_{L}$, since the STRA adjusts the size of channel time values according to $T_{L}$. In contrast to that, the satisfaction factor remains constant when the STRA is not carried out. To better reflect the importance of the proposed mechanism, Figure 30 shows a comparison of the system average Jain's Fairness index for different loads applying or not STRA. It is shown that the overall fairness is much higher when applying STRA and it reaches an approximate of $100 \%$ in the case of lowloaded networks. This is due to the various advantages achieved when applying STRA as it has been previously explained.
In the literature, only few works ([11] and [12]) study the resource allocation for the HR mesh WPAN as previously mentioned. While [11] presents a distributed solution for the mesh WPAN, [12] presents a centralized solution which is the most relevant work for the resource allocation in the HR mesh WPAN. Since the work in [12] studies only the hop-1 MPNCs satisfaction, then the hop-1 algorithm (STRA) is solely applied to perform this comparison. Thus, the performance of the proposed mechanism is compared with that of [12] on the level of the MPNCs satisfaction and fairness index.

For this comparison, the same simulation environment of [12] is considered. Thus, we consider scenarios of 8 , 10 , and 12 MPNCs with a 274,663 superframe duration as studied in [12]. Since the authors in [12] do not study

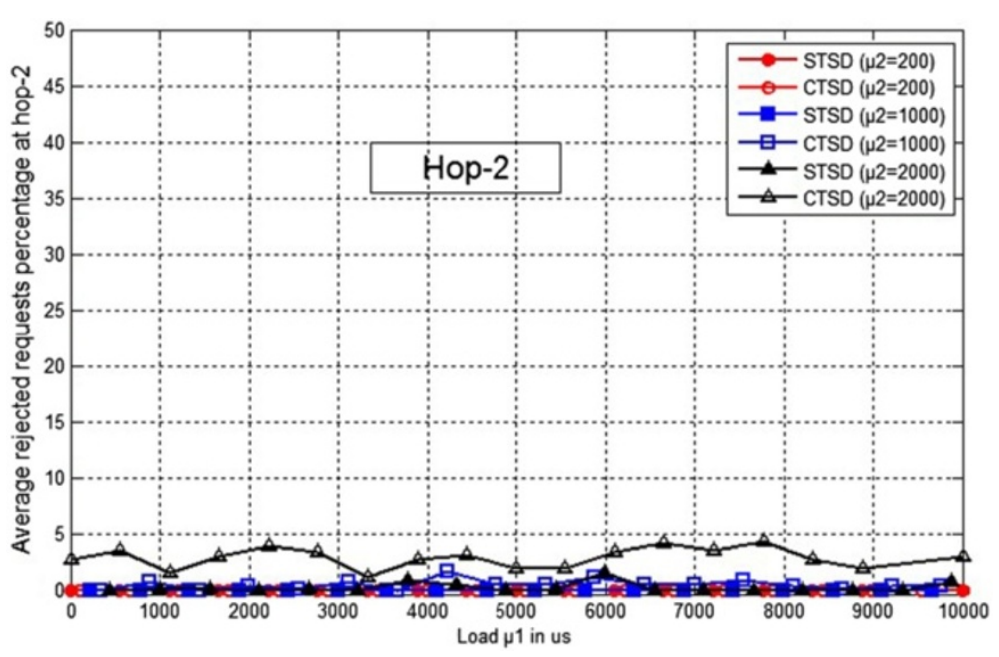

Figure 26 Average rejection rate at hop-2 for different $\mu 1$ and $\mu 2=200,1,000,2,000 \mu$ s. 


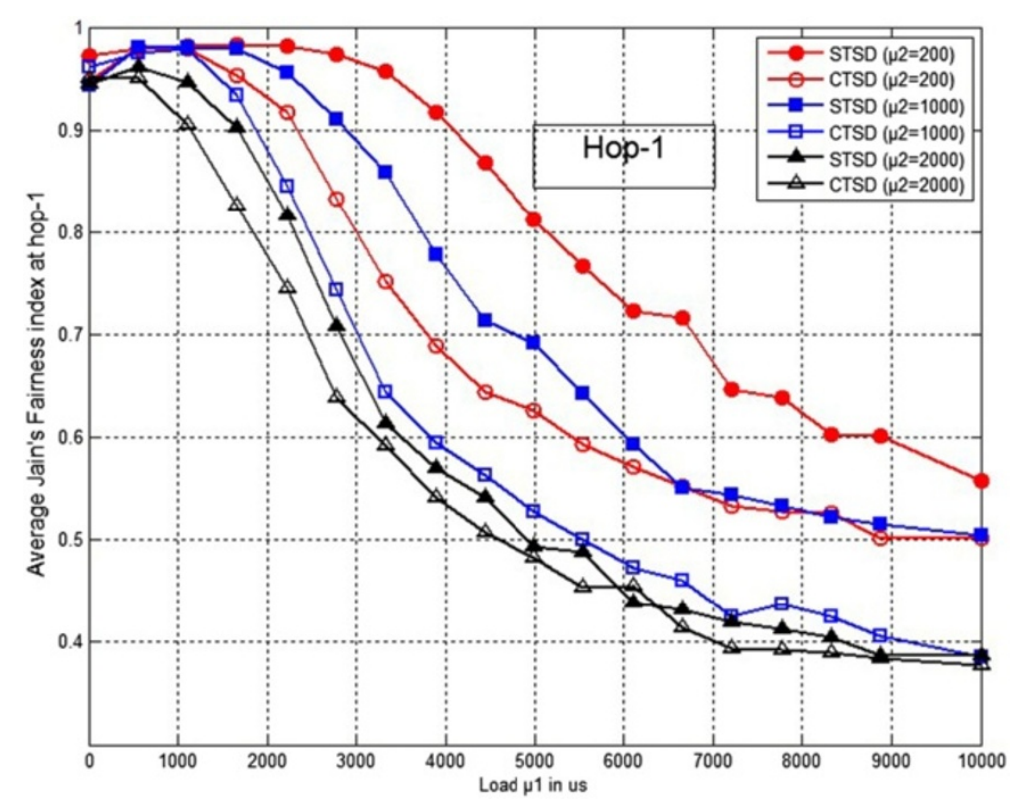

Figure 27 Jain's Fairness index at hop- 1 for different $\mu 1$ and $\mu 2=200,1,000,2,000 \mu$ s.

the satisfaction of the hop-1 MDEVs, only the hop-1 MPNCs satisfaction obtained by running STRA is studied in Figure 31. It is shown that the satisfaction factor achieved in [12] is less than that achieved by STRA for different numbers of MPNCs. Additionally, Figure 32 shows a comparison of the average Jain's Fairness index for the number of MPNCs $(8,9,10$, and 11) studied in [12]. It is also shown that the overall fairness is much higher when applying STRA compared to that obtained in [12].

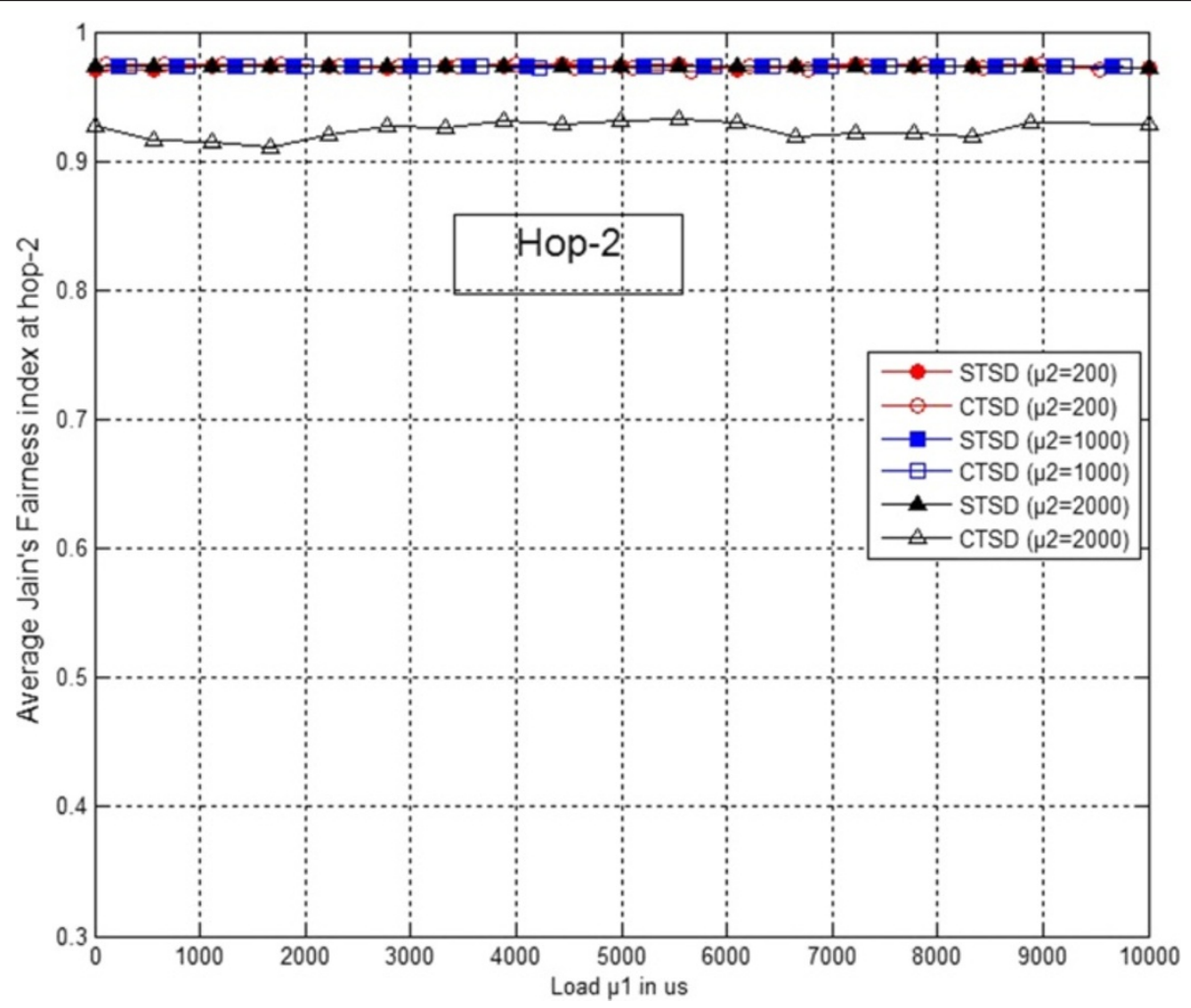

Figure 28 Jain's Fairness index at hop-2 for different $\mu 1$ and $\mu 2=200,1,000,2,000 \mu$ s. 


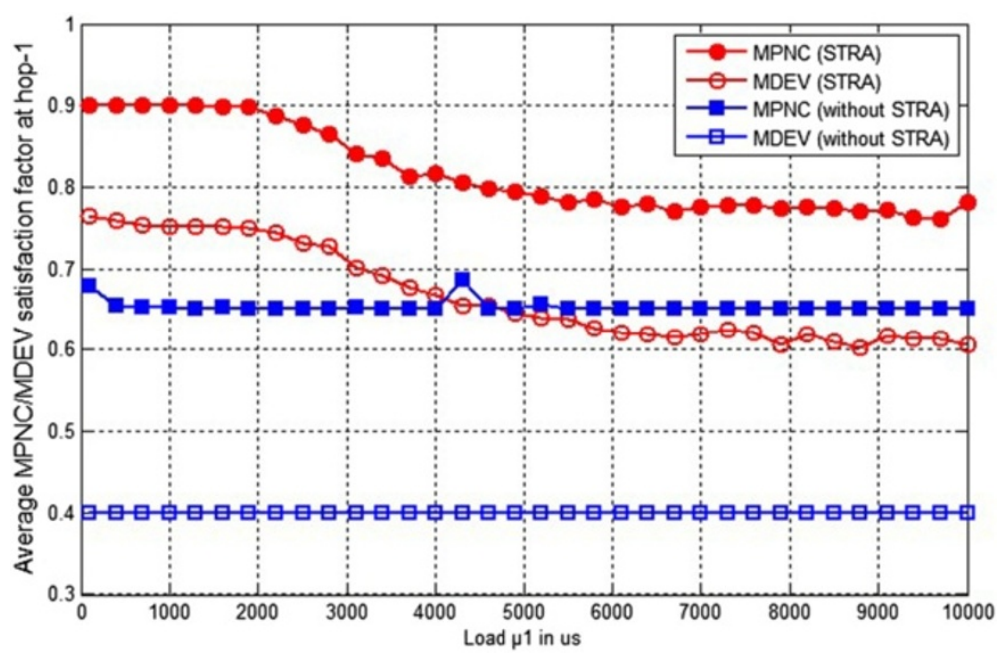

Figure 29 Comparison of average MPNC/MDEV satisfaction with and without applying $T_{L}$ and $P$ (STRA).

For summarizing the obtained results, we identify herein the reasons for obtaining better results using the proposed mechanism in comparison with [12]. These reasons can be described as follows:

Firstly, in addition to the bulk reservation mode, we add the dynamic superframe size, whereas [12] considers the fixed superframe size which could be inefficiently utilized in case of low-loaded networks. Additionally, each MPNC estimates its channel requirements based on a history of previous flows in [12]. Therefore, inefficient superframe utilization can occur because of incorrect channel time estimation for a bulk reservation by each MPNC due to either overestimation or underestimation of the channel time requirement of a cluster.

Secondly, the proposed algorithm considers the channel time requirements of both hop-1 (MPNCs and MDEVs) and hop-2 devices (MDEVs), whereas [12] does not consider the channel time requirements of neither the hop-2 MDEVs in the ref-MPNC cluster nor the hop-2 MDEVs in the hop-1 MPNCs' clusters.

Thirdly, the use of the Flag metric and the priority list assigns higher priority for the devices with a higher number of unserved requests to ascertain that their requests are served in the subsequent superframes. This ensures higher satisfaction and consequently higher fairness among the competing devices compared to [12].

Fourthly, the introduction of the utilization thresholds induces a tradeoff between the satisfaction factor and the rejection rate which directly affects the fairness. This plays an important role in obtaining better fairness using our proposed mechanism compared to [12].

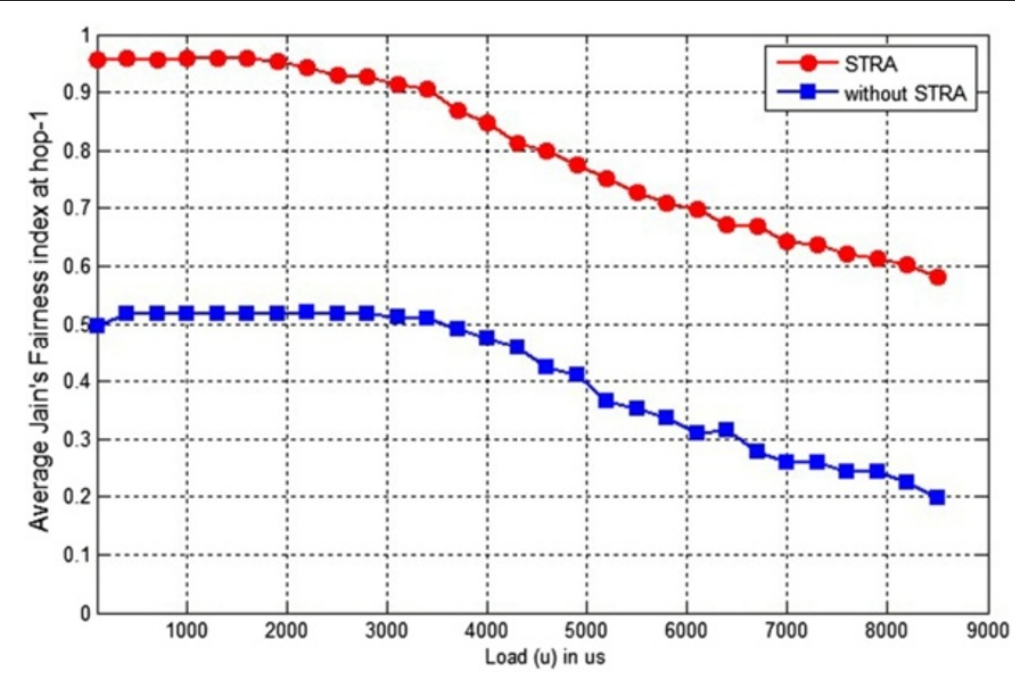

Figure 30 Comparison of Jain's Fairness index with and without applying $T_{L}$ and $P$ (STRA). 


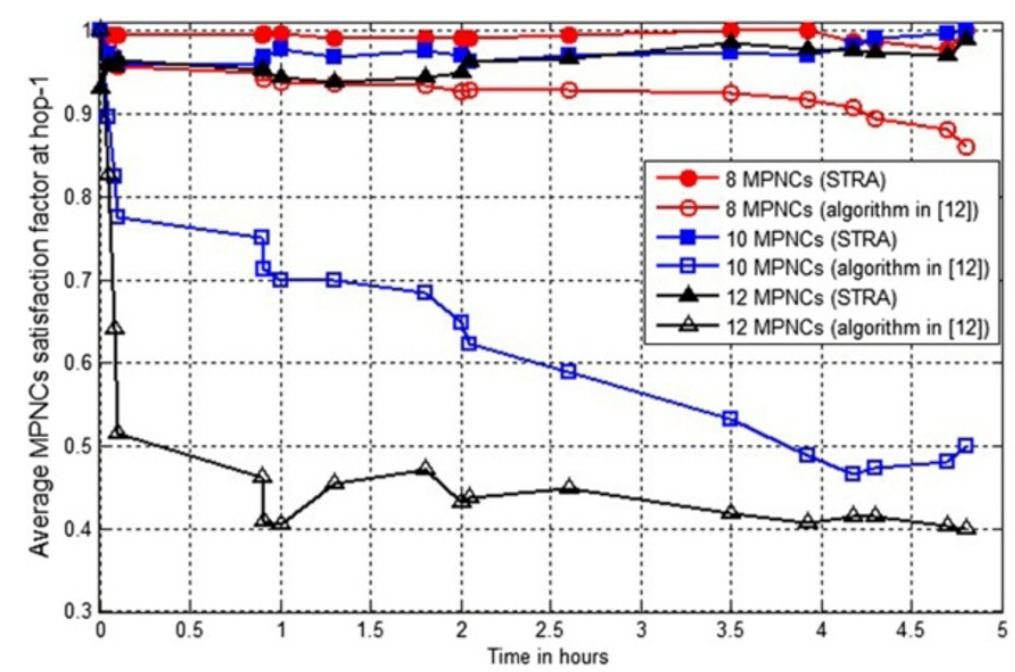

Figure 31 Comparison of average MPNC satisfaction for fixed $\mu 1$ between STRA and algorithm in [12].

Finally, the better results achieved by STRA reflect the advantages of the various new notions that are introduced in this mechanism, namely, the utilization threshold $T_{L}$, the Flag metric, and the priority list. These advantages have already been thoroughly revealed and discussed throughout the various simulation result analyses and the algorithm descriptions.

\section{Conclusions}

In this paper, a two-hop IEEE 802.15.5 resource allocation mechanism, adopting the dynamic superframe size, was proposed. In this mechanism, two algorithms for resource allocation of two-hop IEEE 802.15.5 were defined. A CTAP utilization threshold, a superframe utilization threshold, a Flag metric, and a priority list were introduced.
The conducted simulations illustrated that the superframe is $100 \%$ efficiently utilized when using the proposed mechanism. Additionally, it was demonstrated that this mechanism achieves higher satisfaction and fairness by the use of the utilization thresholds and Flag metric. It was also illustrated that this mechanism prioritizes the MPNCs over the MDEVs at hop- 1 and the RT over NRT MDEVs at hop-2 for a two-hop IEEE 802.15.5. Moreover, comparing the results obtained by the proposed mechanism with other works showed that the proposed mechanism provides better performance on the level of satisfaction factor and fairness.

Finally, the proposed mechanism is fully compliant with the IEEE 802.15.5 standard and is completely relying on the information elements and frames defined in this standard. Thus, this mechanism could be integrated

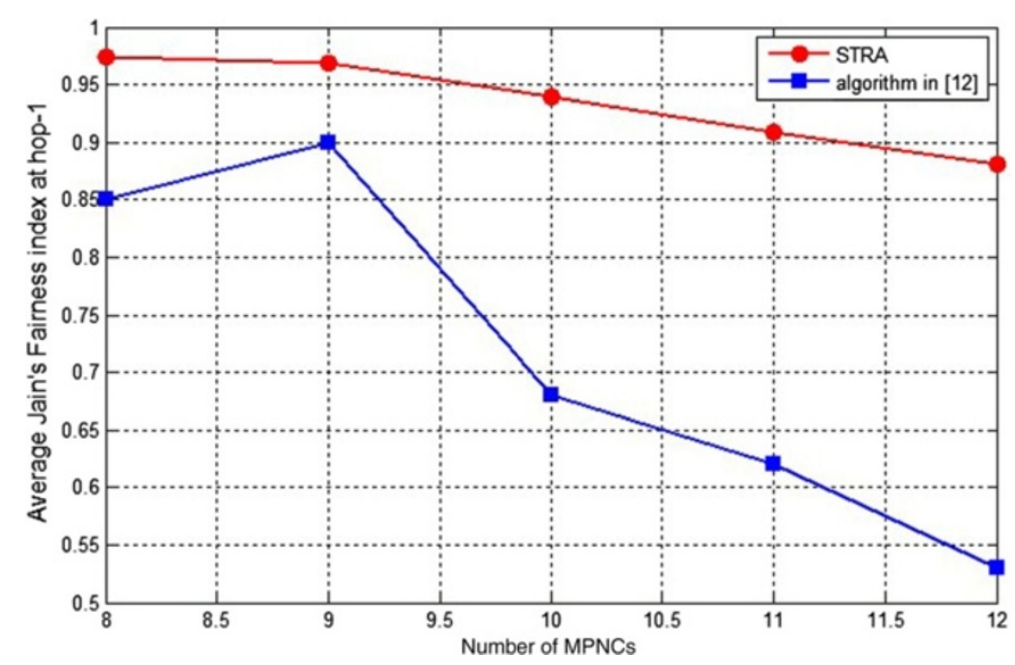

Figure 32 Comparison of Jain's Fairness index for fixed $\mu 1$ between STRA and algorithm in [12]. 
in practice to improve the HR mesh WPAN performance and efficiency as illustrated by the conducted simulation results.

\section{Competing interests}

The authors declare that they have no competing interests.

\section{Author details}

${ }^{1}$ INSA de Rennes, IETR, UMR 6164, F-35708 Rennes, France. ${ }^{2}$ CCE Department, Faculty of Engineering, IUL, 30014 Khaldeh, Lebanon. ${ }^{3}$ Faculty of Engineering, Lebanese University, Rafic Hariri Campus, Hadath, Lebanon.

Received: 4 April 2014 Accepted: 5 November 2014

Published: 23 November 2014

\section{References}

1. IEEE Standard 802.15.3-2003, "Part 15.3: wireless medium access control (MAC and physical layer (PHY) specifications for high rate wireless personal area networks (WPANs), 2003, pp. 1-315

2. SB Jung, S-B Yim, TJ Lee, S-D June, HS Lee, TG Kwon, JW Cho, Multipiconet formation to increase channel utilization in IEEE 802.15.3 high-rate WPAN". Springer-Verlag Lect. Notes Comput. Sci. 3392, 1041-1049 (2006)

3. F Da Costa, "Dynamic Beacon Alignment in Simultaneously Operating Piconets (SOP) Using the Heart Beat Approach," IEEE Standard P802.15-04/135ro, 2004

4. Z Fan, "Multi-hop mesh networking for UWB-based 802.15.3 coverage extension", in Proceedings of the 20th international Conference on Advanced Information Networking and Applications, vol. 1, 2006, pp. 920-925

5. S Jung, H Kim, S Yim, T Lee, "Channel Time Allocation and Routing Algorithm for Multi-hop Communications in IEEE 802.15.3 High-Rate WPAN Mesh Networks", in Proceedings of the 7th international Conference on Computational Science, 2007, pp. 457-465

6. P Xue, P Gong, D Kim, Enhanced IEEE 802.15.3 MAC protocol for efficient support of multiple simultaneously operating piconets. IEEE Trans. Veh. Technol. 57(4), 2548-2559 (2008)

7. S Sindian, A Khalil, A Samhat, M Crussière, J-F Hélard, Resource allocation in high data rate mesh WPAN: a survey paper. Wirel. Pers. Commun. 74(2), 909-932 (2014)

8. S Khan, H Al-Raweshidy, K Sivarajah, Meshed high data rate personal area networks". IEEE Commun. Surv. Tut. 10(1), 58-69 (2008)

9. IEEE Standard 802.15.5-2009, "Part 15.5: Mesh topology capability in Wireless Personal Area Networks (WPANs), 2009, pp. 1-166

10. M Lee, R Zhang, C Zhu, TR Park, C-S Shin, Y-A Jeon, S-H Lee, S-S Choi, Y Liu, S-W Park, Meshing wireless personal area networks: introducing IEEE 802.15.5". IEEE Commun. Mag. 48(1), 54-61 (2010)

11. MS Park, B Lee, SH Rhee, Distributed multiple access control for the wireless mesh personal area networks. Trans. Inform. Syst. E91(2), 258-263 (2008)

12. S Mahmud, S Khan, HS Al-Raweshidy, A resource allocation strategy for meshed high data rate WPANs. IEEE Commun. Lett. 14(6), 524-526 (2010)

13. IEEE Standard 802.15.4-2006, "Part 15.4: Wireless LAN Medium Access Control (MAC) and Physical Layer (PHY) Specifications for Low-Rate Wireless Personal Area Networks (LR-WPANS)", 2006

14. DR Herráiz, A-J García-Sánchez, F García-Sánchez, J García-Haro, On the synchronization of IEEE 802.15.5 wireless mesh sensor networks: shortcomings and improvements". EURASIP J. Wirel. Commun. Netw. 2012(198), 5958-5995 (2012)

15. M Dianati, X Shen, S Naik, "A New Fairness Index for Radio Resource Allocation in Wireless Networks". 5th IEEE Wireless Communications and Networking Conference vol 2, 2005, pp. 712-717

16. A Kumar, J Kleinberg, "Fairness measures for resource allocation", in IEEE Symposium on Foundation of Computer Science, 2000, pp. 568-578

17. R Jain, D Chiu, W Hawe, A quantitative measure of fairness and discrimination for resource allocation in shared computer system. DEC Tech. Rep. 301, (1984)

doi:10.1186/1687-1499-2014-197

Cite this article as: Sindian et al:: Admission control and resource allocation strategies for IEEE 802.15.5. EURASIP Journal on Wireless Communications and Networking 2014 2014:197.

\section{Submit your manuscript to a SpringerOpen ${ }^{\odot}$ journal and benefit from:}

- Convenient online submission

- Rigorous peer review

- Immediate publication on acceptance

- Open access: articles freely available online

- High visibility within the field

- Retaining the copyright to your article

Submit your next manuscript at $\gg$ springeropen.com 